\title{
DYNAMICAL DEGREE, ARITHMETIC ENTROPY, AND CANONICAL HEIGHTS FOR DOMINANT RATIONAL SELF-MAPS OF PROJECTIVE SPACE
}

\author{
JOSEPH H. SILVERMAN
}

\begin{abstract}
Let $\varphi: \mathbb{P}^{N} \rightarrow \mathbb{P}^{N}$ be a dominant rational map. The dynamical degree of $\varphi$ is the quantity $\delta_{\varphi}=\lim \left(\operatorname{deg} \varphi^{n}\right)^{1 / n}$. When $\varphi$ is defined over $\overline{\mathbb{Q}}$, we define the arithmetic degree of a point $P \in \mathbb{P}^{N}(\overline{\mathbb{Q}})$ to be $\alpha_{\varphi}(P)=\lim \sup h\left(\varphi^{n}(P)\right)^{1 / n}$ and the canonical height of $P$ to be $\hat{h}_{\varphi}(P)=\lim \sup \delta_{\varphi}^{-n} n^{-\ell_{\varphi}} h\left(\varphi^{n}(P)\right)$ for an appropriately chosen $\ell_{\varphi}$. In this article we begin by proving some elementary relations and making some deep conjectures relating $\delta_{\varphi}, \alpha_{\varphi}(P), \hat{h}_{\varphi}(P)$, and the Zariski density of the orbit $\mathcal{O}_{\varphi}(P)$ of $P$. We then prove our conjectures for monomial maps.
\end{abstract}

\section{Contents}

1. Introduction 2

2. Relation to earlier work 6

3. The dynamical degree of a rational map 9

4. Arithmetic degree 11

5. Canonical heights for dominant rational maps 13

6. Monomial maps and canonical heights 16

7. Points of canonical height zero for monomial maps 20

8. Proof of Theorem $27 \quad 22$

9. Proof of Corollaries of Theorem $27 \quad 30$

10. Regular affine automorphisms 34

11. Dominant Self-Maps of General Varieties 35

References $\quad 37$

Appendix A. Additional material 40

Date: June 18, 2018.

2010 Mathematics Subject Classification. Primary: 37P30; Secondary: 11G50, $37 \mathrm{~F} 10,37 \mathrm{P} 15$.

Key words and phrases. dynamical degree, arithmetic entropy, canonical height, rational map.

The author's research supported by DMS-0854755. 


\section{INTRODUCTION}

Let $\varphi: \mathbb{P}^{N} \rightarrow \mathbb{P}^{N}$ be a dominant rational map, that is, a map given by homogeneous polynomials $\varphi_{0}, \ldots, \varphi_{N}$ of the same degree having no common nontrivial factors. The map $\varphi$ is called algebraically stable [27] if

$$
\operatorname{deg}\left(\varphi^{n}\right)=(\operatorname{deg} \varphi)^{n} \quad \text { for all } n \geq 1 .
$$

Examples of algebraically stable maps include morphisms and regular affine automorphisms.

In this paper we are principally concerned with the geometry and arithmetic of maps that are not algebraically stable. The (first) $d y$ namical degree of $\varphi$ is defined by

$$
\delta_{\varphi}=\lim _{n \rightarrow \infty}\left(\operatorname{deg}\left(\varphi^{n}\right)\right)^{1 / n}
$$

and $\log \delta_{\varphi}$ is sometimes called the algebraic entropy of $\varphi$; see [12]. The extent to which $\delta_{\varphi}$ differs from $\operatorname{deg}(\varphi)$ is a rough measure of the failure of $\varphi$ to be algebraically stable. Dynamical degrees were initially studied by Russakovskii and Shiffman [53] and Arnol'd [5] in the 1990s, and they have since attracted considerable attention; see for example $[8,10,11,18,22,23,24,26,33,44,43,45,47,61]$. Bellon and Viallet [12] conjectured that $\delta_{\varphi}$ is an algebraic integer, while Hasselblatt and Propp [33] (see also [10]) proved that the sequence $\operatorname{deg}\left(\varphi^{n}\right)$ may be quite irregular in the sense that the power series $\sum_{n \geq 0} \operatorname{deg}\left(\varphi^{n}\right) T^{n}$ need not be a rational function.

The primary objectives of this paper are to study an arithmetic analogue of the dynamical degree and to define an associated canonical height function for dominant rational maps. In this introduction we make a number of conjectures, which we will prove for monomial maps.

So we now assume that $\varphi$ is defined over $\overline{\mathbb{Q}}$, and we consider the iterates of $\varphi$ applied to points in $\mathbb{P}^{N}(\overline{\mathbb{Q}})$. Let

$$
h: \mathbb{P}^{N}(\overline{\mathbb{Q}}) \rightarrow[0, \infty)
$$

denote the usual Weil height; see, e.g., [14, 34, 41, 57, 58] for definitions and basic properties of $h$. An elementary triangle inequality estimate shows that $h\left(\varphi^{n}(P)\right) \ll(\operatorname{deg} \varphi)^{n}$. For points $P \in \mathbb{P}^{N}(\overline{\mathbb{Q}})$ whose orbit $\mathcal{O}_{\varphi}(P)$ is disjoint from the indeterminacy locus $Z(\varphi)$ of $\varphi$, we define the arithmetic degree of $\varphi$ at $P$ to be the quantity

$$
\alpha_{\varphi}(P)=\limsup _{n \rightarrow \infty} h\left(\varphi^{n}(P)\right)^{1 / n} .
$$

We note that since $h(P)$ is, roughly, the information-theoretic content of $P$, it is reasonable to say that $\log \alpha_{\varphi}(P)$ measures the arithmetic 
entropy of the orbit $\mathcal{O}_{\varphi}(P)$. It is not hard to show (Proposition 12) that

$$
\alpha_{\varphi}(P) \leq \delta_{\varphi}
$$

The fact that (1) may be a strict inequality reflects that fact that some orbits capture only a part of the complexity of the map $\varphi$. Our first conjecture describes a sufficient condition for equality.

We set the notation

$$
\mathbb{P}^{N}(\overline{\mathbb{Q}})_{\varphi}=\left\{P \in \mathbb{P}^{N}(\overline{\mathbb{Q}}) \text { such that } \mathcal{O}_{\varphi}(P) \cap Z(\varphi)=\emptyset\right\} \text {. }
$$

We remark that $\mathbb{P}^{N}(\overline{\mathbb{Q}})_{\varphi}$ is Zariski dense in $\mathbb{P}^{N}$, although the proof is not easy; see for example [3].

Conjecture 1. Let $\varphi: \mathbb{P}^{N} \rightarrow \mathbb{P}^{N}$ be a dominant rational map defined over $\overline{\mathbb{Q}}$.

(a) The set

$$
\left\{\alpha_{\varphi}(P): P \in \mathbb{P}^{N}(\overline{\mathbb{Q}})_{\varphi}\right\}
$$

is a finite set of algebraic integers.

(b) Let $P \in \mathbb{P}^{N}(\overline{\mathbb{Q}})_{\varphi}$ be a point such that $\mathcal{O}_{\varphi}(P)$ is Zariski dense in $\mathbb{P}^{N}$. Then $\alpha_{\varphi}(P)=\delta_{\varphi}$.

In Section 7 we prove Conjecture 1 for monomial maps on $\mathbb{P}^{N}$.

As noted earlier, a major objective of this paper is to define and study canonical heights for general dominant rational maps. We recall $[17,57]$ that if $\varphi: \mathbb{P}^{N} \rightarrow \mathbb{P}^{N}$ is a morphism of degree $d \geq 2$, then the canonical height associated to $\varphi$ is the function

$$
\hat{h}_{\varphi}: \mathbb{P}^{N}(\overline{\mathbb{Q}}) \longrightarrow[0, \infty), \quad \hat{h}_{\varphi}(P)=\lim _{n \rightarrow \infty} \frac{1}{d^{n}} h\left(\varphi^{n}(P)\right) .
$$

The canonical height for a morphism is characterized by the properties

$$
\hat{h}_{\varphi}(P)=h(P)+O(1) \quad \text { and } \quad \hat{h}_{\varphi}(\varphi(P))=d \hat{h}_{\varphi}(P),
$$

from which one easily deduces that

$$
P \in \operatorname{PrePer}(\varphi) \Longleftrightarrow \hat{h}_{\varphi}(P)=0 .
$$

For general dominant rational maps we have $\operatorname{deg}\left(\varphi^{n}\right) \approx \delta_{\varphi}^{n}$, so it is natural to look at

$$
\frac{1}{\delta_{\varphi}^{n}} h\left(\varphi^{n}(P)\right),
$$

but the approximation $\operatorname{deg}\left(\varphi^{n}\right) \approx \delta_{\varphi}^{n}$ is insufficiently precise. For example, the map $\varphi(x, y)=\left(x^{d} y, y^{d}\right)$ satisfies $\operatorname{deg}\left(\varphi^{n}\right)=d^{n}+n d^{n-1}$, so $\operatorname{deg}\left(\varphi^{n}\right)$ grows faster than $\delta_{\varphi}^{n}=d^{n}$. This leads us to make the following conjecture (cf. [33]), which will provide the required correction factor. 
Conjecture 2. Let $\varphi: \mathbb{P}^{N} \rightarrow \mathbb{P}^{N}$ be a dominant rational map. Then the infimum

$$
\ell_{\varphi}=\inf \left\{\ell \geq 0: \sup _{n \geq 1} \frac{\operatorname{deg}\left(\varphi^{n}\right)}{n^{\ell} \delta_{\varphi}^{n}}<\infty\right\}
$$

exists and is an integer satisfying $0 \leq \ell_{\varphi} \leq N$.

We note that Conjecture 2 is really three conjectures, first that $\delta_{\varphi}^{-n} \operatorname{deg}\left(\varphi^{n}\right)$ grows at most polynomially in $n$, second that the growth rate is essentially $n^{\ell}$ for an integer $\ell$, and third that $\ell$ is between 0 and $N$. As noted by the referee, a bold person might even conjecture that $\operatorname{deg}\left(\varphi^{n}\right) \asymp n^{\ell} \delta_{\varphi}^{n}$, which would preclude for example the appearence of powers of $\log n$ in the growth rate. See Section 2.1 for a further discussion of Conjecture 2 .

Definition. With notation as above, the canonical height of $P \in$ $\mathbb{P}^{N}(\overline{\mathbb{Q}})_{\varphi}$ with respect to $\varphi$ is

$$
\hat{h}_{\varphi}(P)=\limsup _{n \rightarrow \infty} \frac{1}{n^{\ell \varphi} \delta_{\varphi}^{n}} h\left(\varphi^{n}(P)\right) .
$$

We note that the limsup is necessary, since it is easy to construct examples for which the limit diverges by oscillation; see Example 14. Also, it is easy to check (Proposition 19) that

$$
\hat{h}_{\varphi}(\varphi(P))=\delta_{\varphi} \hat{h}_{\varphi}(P) .
$$

If $\delta_{\varphi}>1$, we suspect that $\hat{h}_{\varphi}(P)$ is finite, and we prove that this holds for monomial maps (Proposition 25). However, if $\delta_{\varphi}=1$, then it is possible to have $\ell_{\varphi} \geq 1$ and $\hat{h}_{\varphi}(P)=\infty$, as we show in Example 17 .

It is not hard to prove that

$$
\hat{h}_{\varphi}(P)>0 \quad \Longrightarrow \quad \alpha_{\varphi}(P)=\delta_{\varphi} ;
$$

see Proposition 19(d). The converse to (2) is not true in general; see the discussion before the statement of Corollary 33. It would be very interesting to find general geometric conditions on $\varphi$ that imply the converse of (2). We prove in Corollary 33 that the converse holds for monomial maps associated to diagonalizable matrices.

A fundamental property of the canonical height for morphisms is that height zero characterizes points with finite orbit. (N.B. We always work over $\overline{\mathbb{Q}}$. The situation over function fields is subtler; see for example $[7$, 13].) For any dominant rational map $\varphi$ with $\delta_{\varphi}>1$ or $\ell_{\varphi}>0$, we clearly have

$$
P \in \operatorname{PrePer}(\varphi) \quad \Longrightarrow \quad \hat{h}_{\varphi}(P)=0
$$

but the converse is not true in general, since there may be subvarieties on which $\varphi$ acts via lower degree. This leads to the following conjecture. 
Conjecture 3. Let $\varphi: \mathbb{P}^{N} \rightarrow \mathbb{P}^{N}$ be a dominant rational map defined over $\overline{\mathbb{Q}}$ with dynamical degree $\delta_{\varphi}>1$, and let $P \in \mathbb{P}^{N}(\overline{\mathbb{Q}})_{\varphi}$ be a point whose orbit $\mathcal{O}_{\varphi}(P)$ is Zariski dense in $\mathbb{P}^{N}$. Then $\hat{h}_{\varphi}(P)>0$.

We observe that Conjecture 3 and the elementary implication (2) imply Conjecture $1(\mathrm{~b})$.

The main theorem in this paper (Theorem 27) gives a geometric description of the set of points satisfying $\hat{h}_{\varphi}(P)=0$ for monomial maps $\varphi$. Immediate corollaries include proofs of Conjectures 1 and 3 for monomial maps. We also note that a strong form of Conjecture 2 is true for monomial maps; this was proven independently by Lin [44] and Jonsson and Wulcan [36].

We recall that a monomial map is an endomorphism of the torus $\mathbb{G}_{m}^{N}$, i.e., a map

$$
\varphi_{A}: \mathbb{G}_{m}^{N} \longrightarrow \mathbb{G}_{m}^{N}
$$

of the form

$$
\varphi_{A}\left(X_{1}, \ldots, X_{N}\right)=\left(X_{1}^{a_{11}} X_{2}^{a_{12}} \cdots X_{N}^{a_{1 N}}, \ldots, X_{1}^{a_{N 1}} X_{2}^{a_{N 2}} \cdots X_{N}^{a_{N N}}\right),
$$

where $A=\left(a_{i j}\right)$ is an $N$-by- $N$ matrix with integer coefficients. The associated rational map $\varphi_{A}: \mathbb{P}^{N} \rightarrow \mathbb{P}^{N}$ is $\operatorname{dominant}$ if $\operatorname{det}(A) \neq 0$. Hasselblatt and Propp [33] have shown that the dynamical degree of $\varphi_{A}$ is equal to the spectral radius of $A$, i.e., the magnitude of the largest eigenvalue of $A$.

The following is a special case of our main theorem and its corollaries; see Section 7 for details.

Theorem 4. Conjectures 1 and 3 are true for monomial maps. More precisely, let $\varphi_{A}$ be a monomial map with $\delta_{\varphi_{A}}>1$.

(a) The arithmetic degrees of $\varphi_{A}$ satisfy

$$
\left\{\alpha_{\varphi_{A}}(P): P \in \mathbb{G}_{m}^{N}(\overline{\mathbb{Q}})\right\} \subset\{\text { eigenvalues of } A\} \text {. }
$$

In particular, $\alpha_{\varphi_{A}}(P)$ is an algebraic integer for all $P \in \mathbb{G}_{m}^{N}(\overline{\mathbb{Q}})$.

(b) Let $P \in \mathbb{G}_{m}^{N}(\overline{\mathbb{Q}})$ be a point with $\hat{h}_{\varphi_{A}}(P)=0$. Then $\mathcal{O}_{\varphi}(P)$ is contained in a proper (possibly disconnected) algebraic subgroup of $\mathbb{G}_{m}^{N}$.

(c) If the matrix $A$ is diagonalizable over $\mathbb{C}$, then

$$
\hat{h}_{\varphi}(P)=0 \Longleftrightarrow \alpha_{\varphi}(P)<\delta_{\varphi} .
$$

(d) If the characteristic polynomial of the matrix $A$ is irreducible over $\mathbb{Q}$, then

$$
\hat{h}_{\varphi}(P)=0 \Longleftrightarrow \mathcal{O}_{\varphi_{A}}(P) \text { is finite. }
$$


Theorem 4 is proven in Section 9 as a series of corollaries to Theorem 27, which is our main result. The proof of Theorem 27 uses a compactness argument, the product formula, Baker's theorem on linear-forms-in-logarithms, and a lot of linear algebra. In particular, Baker's theorem is needed to show the $\operatorname{Gal}(\overline{\mathbb{Q}} / \mathbb{Q})$-invariance of the set of $\overline{\mathbb{Q}}$-linear relations on a set of log absolute values

$$
\log \left\|x_{1}\right\|_{v}, \ldots, \log \left\|x_{N}\right\|_{v}
$$

where the $x_{i}$ are in $\overline{\mathbb{Q}}$ and $v \in M_{\overline{\mathbb{Q}}}$ is an (archimedean) absolute value on $\overline{\mathbb{Q}}$.

We conclude the paper with two additional results. In Section 10 we use Kawaguchi's theory of canonical heights for regular affine automorphisms to prove our conjectures for maps of this type, and in Section 11 we generalize Conjecture 1 to dominant rational self-maps of arbitrary (nonsingular) varieties and prove that it is true for automorphisms of certain K3 surfaces.

Addendum. While this paper was under review, a number of authors have written papers that grew out of the questions raised and results proven in this paper. We mention in particular a paper of Jonsson and Wulcan [37] in which they prove much of Conjectures 1 and 3 for polynomial morphisms $\varphi: \mathbb{A}^{2} \rightarrow \mathbb{A}^{2}$ of small topological degree, and a paper of Kawaguchi and the author [40] in which it is shown that $\alpha_{\varphi}(P) \leq \delta_{\varphi}$ holds for dominant rational self-maps of (normal) varieties.

Remark. In the ArXiv version of this article, for the convenience of the reader we have an included an appendix giving further details about various elemenatry remarks and assertions. The appendix will not appear in the published version.

Acknowledgements. The author thanks Charles Favre, Mattias Jonsson, Shu Kawaguchi, Jan-Li Lin, James Propp, Juan Rivera-Letelier, and Tom Ward for their helpful comments on the initial draft. The author also thanks Mattias Jonsson for pointing out that the converse to (2) does not hold in general, Jan-Li Lin for showing the author the short proof of Lemma 22 (which improved the original proof that worked only over $\overline{\mathbb{Q}}$ ), and the referee for his careful reading of the manuscript and his/her many suggestions, including especially a simplification and generalization of the proof of Theorem 27 that eliminated the assumption that the matrix $A$ be diagonalizable.

\section{RELATION TO EARLIER WORK}


2.1. Growth rate of $\operatorname{deg}\left(\varphi^{n}\right)$. Conjecture 2 is related to questions raised by Hasselblatt and Propp [33]. In particular, they ask [33, Question 9.5] if the degree sequence $\operatorname{deg}\left(\varphi^{n}\right)$ can be simultaneously subexponential and superpolynomial. Conjecture 2 says that this cannot happen. They further ask [33, Question 9.6] if, whenever $\operatorname{deg}\left(\varphi^{n}\right)$ is bounded by a power of $n$, must it grow essentially like $n^{\ell}$ for a nonnegative integer $\ell$. Conjecture 2 says that this is true, so for example a growth rate of order $\sqrt{n}$ should not be possible.

The classification results of Diller and Favre [22] can be used to show that Conjecture 2 is true for birational maps of $\mathbb{P}^{2}$ having $\delta_{\varphi}=1$. See also [11] for families of birational maps on $\mathbb{P}^{2}$ having $\delta_{\varphi}=1$ and $\ell_{\varphi}=2$, which shows that $\ell_{\varphi}$ may be as large as the dimension. Lin [44] and Jonsson and Wulcan [36] have shown that a strong form of Conjecture 2 holds for monomial maps; see Theorem 24. See also [15, 24, 25] for a proof that Conjecture 2 holds for certain rational maps of $\mathbb{P}^{2}$, including in particular all polynomial maps of $\mathbb{A}^{2}$.

In general, it is very difficult to compute, or even to estimate, the value of the dynamical degree of a rational map in dimension greater than 2 , since even on a computer one generally cannot compute the map $\varphi^{n}$ for moderate values of $n$. See $[4,8,9]$ for some discussion of these issues and for the computation of $\delta_{\varphi}$ for certain higher-dimensional maps.

\subsection{Canonical heights for regular affine automorphisms.}

The theory of canonical heights for morphisms of $\mathbb{P}^{N}$ is well known and may be developed exactly as was done by Néron and Tate in their theory of canonical heights on abelian varieties; see for example [17] or [57, §3.4]. A regular affine automorphism [54] is an automorphism $\varphi: \mathbb{A}^{N} \rightarrow \mathbb{A}^{N}$ whose extension to a rational map $\varphi: \mathbb{P}^{N} \rightarrow \mathbb{P}^{N}$ satisfies $Z(\varphi) \cap Z\left(\varphi^{-1}\right)=\emptyset$. Regular affine automorphisms are algebraically stable, i.e., $\delta_{\varphi}=\operatorname{deg}(\varphi)$; see [54, Chapter 2]. Shu Kawaguchi has developed a theory of canonical heights for such maps. Kawaguchi's construction is described in [38] and [57, Exercises 7.17-7.22], and the subtle height inequality needed to justify the construction is given in [39] and [42]. There is thus a satisfactory theory of canonical heights for regular affine automorphisms, and the present article may be viewed as a first step towards establishing an analogous theory for general dominant rational maps.

2.3. The dynamical Manin-Mumford conjecture. The relationship between preperiodic points and canonical heights, and in particular Conjecture 3, may have some bearing on the not-yet-precisely-formulated dynamical Manin-Mumford conjecture. A naive conjecture, 
modeled after Zhang's conjecture for polarized morphisms, might say the following: Let $\varphi: \mathbb{P}^{N} \rightarrow \mathbb{P}^{N}$ be a dominant rational map with $\delta_{\varphi}>1$, and let $X \subset \mathbb{P}^{N}$ be an irreducible subvariety. If $\operatorname{PrePer}(\varphi) \cap X$ is Zariski dense in $X$, then $X$ is preperiodic. This naive statement is clearly false. For example, let $\varphi: \mathbb{P}^{2} \rightarrow \mathbb{P}^{2}$ be $\varphi([x, y, z])=$ $\left[x^{2} z, y^{3}, z^{3}\right]$ and take $X=\{x=y\}$. But some carefully formulated dynamical Manin-Mumford statements have been proven; see for example [28, 29]. Our hope is that the existence of a canonical height characterizing preperiodic points as being exactly those points having height zero might be a helpful tool for proving Manin-Mumford type results for more general maps.

2.4. Integrability and arithmetic entropy. The relationship between the degree growth of iterates of a rational map and the existence of invariant fibrations or more general geometric invariant structures is an area of intense activity in both the mathematical and the physics literatures. When an invariant structure of a specified type exists, one often says that the map is integrable, although there is not yet a precise general definition of integrability. We refer the reader to [31] for a survey on integrability of discrete dynamical systems and for some (heuristic) methods of detecting integrability, including studying the cycle structure of the reduction of $\varphi$ acting on $\mathbb{P}^{N}\left(\mathbb{F}_{q}\right)$ for varying finite fields $\mathbb{F}_{q}[50,51,52]$ and studying the growth rate of $h\left(\varphi^{n}(P)\right)$ for rational or algebraic points $P[1,32,35]$. In particular, Halburd [32] defines a map $\varphi$ to be Diophantine integrable if $h\left(\varphi^{n}(P)\right)$ grows no faster than polynomially in $n$ for all rational (or all algebraic) points $P$. In our terminology, such orbits have arithmetic degree 1, equivalently, arithmetic entropy 0. We also mention Buium's beautiful arithmetic characterization [16] of Lattès maps (one-dimensional integrable maps) in terms of their mod $p$ reductions.

2.5. Another type of algebraic entropy. There is another notion of algebraic entropy defined for self-maps of topological groups with various additional structures, e.g., for locally compact abelian groups. See for example the papers $[2,30,21,49,48,60]$. In particular, the paper [30] shows that the algebraic entropy of an endomorphism of a finite-dimensional rational vector space is the Mahler measure of the characteristic polynomial of the associated matrix, which is similar to results of Hasselblatt-Propp [33] and Lin [43, 44]. 


\section{The Dynamical Degree of a RAtional MAP}

Let $\varphi: \mathbb{P}^{N} \rightarrow \mathbb{P}^{N}$ be a dominant rational map of degree $d$. If $\varphi$ is a morphism, then the degree of $\varphi^{n}$ is simply $d^{n}$, but in general the degree of $\varphi^{n}$ may be strictly smaller than $d^{n}$. The sequence of degrees $\left(\operatorname{deg} \varphi^{n}\right)_{n \geq 1}$ is both interesting and often surprisingly difficult to analyze.

Definition. Let $\varphi: \mathbb{P}^{N} \rightarrow \mathbb{P}^{N}$ be a dominant rational map. The (first) dynamical degree of $\varphi$ is the quantity

$$
\delta_{\varphi}=\lim _{n \rightarrow \infty}\left(\operatorname{deg} \varphi^{n}\right)^{1 / n} .
$$

Example 5. The iterates of the map

$$
\varphi([x, y, z])=\left[y z, x y, z^{2}\right]
$$

are easily computed to be

$$
\varphi^{n}([x, y, z])=\left[x^{F_{n-1}} y^{F_{n}} z^{F_{n}}, x^{F_{n}} y^{F_{n+1}}, z^{F_{n+2}}\right],
$$

where $F_{n}$ is the $n$ 'th Fibonacci number. Hence

$$
\delta_{\varphi}=\lim _{n \rightarrow \infty} F_{n+2}^{1 / n}=\frac{1+\sqrt{5}}{2}
$$

is the golden ratio. We remark that $\varphi$ is birational and regular, i.e., satisfies $Z(\varphi) \cap Z\left(\varphi^{-1}\right)=\emptyset$, but it is not an affine automorphism.

Example 6. The map

$$
\varphi: \mathbb{A}^{3} \longrightarrow \mathbb{A}^{3}, \quad \varphi(x, y, z)=(y, z, x+y z),
$$

is an affine automorphism, but it is not regular, since $Z(\varphi) \cap Z\left(\varphi^{-1}\right)$ is a line. An easy induction shows that $\operatorname{deg}\left(\varphi^{n}\right)=F_{n}$, so this map also has $\delta_{\varphi}=\frac{1+\sqrt{5}}{2}$.

For the convenience of the reader, we recall the proof of the following well-known properties of the dynamical degree.

Proposition 7. The limit defining the dynamical degree exists and satisfies

$$
\delta_{\varphi}=\inf _{n \geq 1}\left(\operatorname{deg} \varphi^{n}\right)^{1 / n} .
$$

Proof. We note that for any rational maps $\varphi, \psi: \mathbb{P}^{N} \rightarrow \mathbb{P}^{N}$, we have

$$
\operatorname{deg}(\varphi \circ \psi) \leq(\operatorname{deg} \varphi)(\operatorname{deg} \psi) .
$$

To ease notation, we let

$$
d_{n}=\log \operatorname{deg}\left(\varphi^{n}\right) .
$$


We need to prove that the sequence $d_{n} / n$ converges and is equal to its infimum. From (3) we see that

$$
d_{i+j} \leq d_{i}+d_{j} \text { for all } i \text { and } j .
$$

Fix an integer $m$ and write $n=m q+r$ with $0 \leq r<m$. Then

$$
\frac{d_{n}}{n}=\frac{d_{m q+r}}{n} \leq \frac{q d_{m}+d_{r}}{n}=\frac{d_{m}}{m} \cdot \frac{1}{1+r / m q}+\frac{d_{r}}{n} \leq \frac{d_{m}}{m}+\frac{d_{r}}{n} .
$$

Now take the limsup as $n \rightarrow \infty$, keeping in mind that $m$ is fixed and $r<m$, so $d_{r}$ is bounded. This gives

$$
\limsup _{n \rightarrow \infty} \frac{d_{n}}{n} \leq \frac{d_{m}}{m}
$$

Taking the infimum over $m$ shows that

$$
\limsup _{n \rightarrow \infty} \frac{d_{n}}{n} \leq \inf _{m \geq 1} \frac{d_{m}}{m} \leq \liminf _{m \rightarrow \infty} \frac{d_{m}}{m},
$$

and hence all three quantities must be equal.

The dynamical degrees in Examples 5 and 6 are the golden ratio, which is an algebraic integer. This is a consequence of the fact that their degree sequences $\left(\operatorname{deg} \varphi^{n}\right)_{n \geq 1}$ satisfy a linear recurrence with constant coefficients. It turns out that not all degree sequences satisfy such linear recurrences. For example, it is shown in [33] that the degree sequence for the map $\varphi(x, y)=\left(x y^{2}, x^{-2} y\right)$ does not satisfy a linear recurrence with constant coefficients, although it is still true that $\delta_{\varphi}$ is an algebraic integer for this map.

Conjecture 8. (Bellon-Viallet [12]) Let $\varphi: \mathbb{P}^{N} \rightarrow \mathbb{P}^{N}$ be a dominant rational map defined over $\mathbb{C}$. Then its dynamical degree $\delta_{\varphi}$ is an algebraic integer.

Example 9. If $\delta_{\varphi}>1$, then $\operatorname{deg}\left(\varphi^{n}\right)$ grows roughly like $\delta_{\varphi}^{n}$. One might ask if the growth rates are the same, but it can happen that the ratio $\left(\operatorname{deg} \varphi^{n}\right) / \delta_{\varphi}^{n}$ grows like a power of $n$. For example, let $d \geq 2$ be an integer, and let $\varphi: \mathbb{P}^{N} \rightarrow \mathbb{P}^{N}$ be the dominant rational map given in affine coordinates by

$$
\varphi=\left(X_{1}^{d} X_{2}, X_{2}^{d} X_{3}, X_{3}^{d} X_{4}, \cdots, X_{N-1}^{d} X_{N}, X_{N}^{d}\right) .
$$

(This is an example of a monomial map; see Section 6.) It is easy to prove that

$$
\operatorname{deg}\left(\varphi^{n}\right)=d^{n}+n d^{n-1}+\left(\begin{array}{l}
n \\
2
\end{array}\right) d^{n-2}+\cdots+\left(\begin{array}{c}
n \\
N-1
\end{array}\right) d^{n-N+1} .
$$


Thus

$$
\lim _{n \rightarrow \infty} \frac{\operatorname{deg}\left(\varphi^{n}\right)}{d^{n} \cdot n^{N-1}}=\frac{1}{(N-1) ! d^{N-1}},
$$

so Conjecture 2 is true for this map with $\delta_{\varphi}=d$ and $\ell_{\varphi}=N-1$. Trivial modifications of this example give maps with $\delta_{\varphi}=d$ and with $\ell_{\varphi}$ equal to any integer between 0 and $N-1$.

Remark 10. We remark that more generally, a dominant rational map $\varphi: \mathbb{P}^{N} \rightarrow \mathbb{P}^{N}$ has $N$ different associated dynamical degrees corresponding to its action on linear subspaces of various dimensions. Thus the $k^{\text {th }}$ dynamical degree of $\varphi$ is the quantity

$$
\delta_{k, \varphi}=\limsup _{n \rightarrow \infty}\left(\operatorname{deg}\left(\varphi^{n}\right)^{*} L\right)^{1 / n}
$$

where $L \subset \mathbb{P}^{N}$ is a generic linear subvariety of codimension $k$. These dynamical degrees were introduced in [53], and are computed for monomial maps in $[26,43]$.

For further material on the dynamical degree, see for example $[5,8$, $11,12,18,19,22,23,24,26,33,44,43,45,47,53,59]$

\section{Arithmetic Degree}

If $\varphi: \mathbb{P}^{N} \rightarrow \mathbb{P}^{N}$ is a morphism of degree $d \geq 2$ defined over $\overline{\mathbb{Q}}$ and $P \notin \operatorname{PrePer}(\varphi)$, then $h\left(\varphi^{n}(P)\right)$ grows like a multiple of $d^{n}$, so in particular $h\left(\varphi^{n}(P)\right)^{1 / n} \rightarrow d$ as $n \rightarrow \infty$. If $\varphi$ is a rational map, but not a morphism, then $h\left(\varphi^{n}(P)\right)$ may grow more slowly than $d^{n}$, which suggests (by analogy with dynamical degree) the following definition.

Definition. Let $\varphi: \mathbb{P}^{N} \rightarrow \mathbb{P}^{N}$ be a dominant rational map defined over $\overline{\mathbb{Q}}$, and let $P \in \mathbb{P}^{N}(\overline{\mathbb{Q}})_{\varphi}$. The arithmetic degree of $\varphi$ at $P$ is the quantity

$$
\alpha_{\varphi}(P)=\limsup _{n \rightarrow \infty} h\left(\varphi^{n}(P)\right)^{1 / n} .
$$

(If $h\left(\varphi^{n}(P)\right)=0$ for all sufficiently large $n$, which can only happen if $P \in \operatorname{PrePer}(\varphi)$, then by convention we set $\alpha_{\varphi}(P)=1$.)

Example 11. Consider the map given in affine coordinates by

$$
\varphi(x, y, z)=\left(x y, y, z^{2}\right) .
$$

Then

$$
\varphi^{n}(x, y, z)=\left(x y^{n}, y, z^{2^{n}}\right)
$$


so $\delta_{\varphi}=2$, since the $z$-coordinate dominates the degree of $\varphi^{n}$. However, if we consider a point of the form $P=(x, y, \zeta)$ with $\zeta$ a root of unity, then

$$
h\left(\varphi^{n}(x, y, \zeta)\right) \leq h(x)+n h(y)
$$

so $\alpha_{\varphi}(P)=1$. Thus $\alpha_{\varphi}(P)=1$ on a countable union of two-dimensional hyperplanes of $\mathbb{P}^{3}$. These hyperplanes are preperiodic for $\varphi$.

We now show that $\alpha_{\varphi}(P) \leq \delta_{\varphi}$.

Proposition 12. Let $\varphi: \mathbb{P}^{N} \rightarrow \mathbb{P}^{N}$ be a dominant rational map of degree $d \geq 2$ defined over $\overline{\mathbb{Q}}$, and let $P \in \mathbb{P}^{N}(\overline{\mathbb{Q}})_{\varphi}$. Then

$$
\alpha_{\varphi}(P) \leq \delta_{\varphi}
$$

Proof. A standard triangle inequality estimate says that

$$
h(\varphi(Q)) \leq d h(Q)+O_{\varphi}(1) .
$$

We start with a telescoping sum inequality computation.

$$
\begin{aligned}
h\left(\varphi^{n}(P)\right)-d^{n} h(P) & =\sum_{i=1}^{n} d^{n-i}\left[h\left(\varphi^{i}(P)\right)-d h\left(\varphi^{i-1}(P)\right)\right] \\
& \leq \sum_{i=1}^{n} d^{n-i} \sup _{Q \in \mathbb{P}^{N}(\overline{\mathbb{Q}})}\{h(\varphi(Q))-d h(Q)\} \\
& \leq \sum_{i=1}^{n} d^{n-i} O_{\varphi}(1) \quad \text { from }(4), \\
& =O_{\varphi}\left(d^{n}\right) .
\end{aligned}
$$

Thus there is a constant $C(\varphi, P)$, depending as indicated on $\varphi$ and $P$, such that

$$
h\left(\varphi^{n}(P)\right) \leq C(\varphi, P)(\operatorname{deg} \varphi)^{n} \quad \text { for all } n \geq 1 .
$$

For each integer $k \geq 1$, we write $d_{k}=\operatorname{deg}\left(\varphi^{k}\right)$. Applying (5) to the $\operatorname{map} \varphi^{k}$ yields

$$
h\left(\varphi^{n k}(P)\right) \leq C\left(\varphi^{k}, P\right)\left(\operatorname{deg} \varphi^{k}\right)^{n}
$$

Hence

$$
\limsup _{n \rightarrow \infty} h\left(\varphi^{n k}(P)\right)^{1 / n k} \leq \limsup _{n \rightarrow \infty} C\left(\varphi^{k}, P\right)^{1 / n k}\left(\operatorname{deg} \varphi^{k}\right)^{1 / k}=\left(\operatorname{deg} \varphi^{k}\right)^{1 / k}
$$

We next show that $\alpha_{\varphi}(P)$ can be computed using the subsequence of iterates $\left(\varphi^{n k}\right)_{n \geq 1}$. To see this, we estimate

$$
\alpha_{\varphi}(P)=\limsup _{m \rightarrow \infty} h\left(\varphi^{m}(P)\right)^{1 / m}
$$




$$
\begin{aligned}
& =\limsup _{n \rightarrow \infty} \max _{0 \leq i<k} h\left(\varphi^{n k+i}(P)\right)^{1 /(n k+i)} \\
& \leq \limsup _{n \rightarrow \infty} \max _{0 \leq i<k}\left(d^{i} h\left(\varphi^{n k}(P)\right)+O\left(d^{i}\right)\right)^{1 /(n k+i)} \quad \text { from }(4) \\
& \leq \limsup _{n \rightarrow \infty}\left(d^{k-1} h\left(\varphi^{n k}(P)\right)+O\left(d^{k}\right)\right)^{1 / n k} \\
& =\limsup _{n \rightarrow \infty} h\left(\varphi^{n k}(P)\right)^{1 / n k} \\
& \leq \alpha_{\varphi}(P) \text { by definition of } \alpha_{\varphi}(P) .
\end{aligned}
$$

This proves that for any integer $k \geq 2$, the arithmetic degree of $P$ can be computed as

$$
\alpha_{\varphi}(P)=\limsup _{n \rightarrow \infty} h\left(\varphi^{n k}(P)\right)^{1 / n k} .
$$

Combining (6) and (7) gives

$$
\alpha_{\varphi}(P) \leq\left(\operatorname{deg} \varphi^{k}\right)^{1 / k}
$$

This estimate holds for all $k \geq 1$, so letting $k \rightarrow \infty$ gives the desired result $\alpha_{\varphi}(P) \leq \delta_{\varphi}$

Question 13. If $P \in \operatorname{PrePer}(\varphi)$, then $\alpha_{\varphi}(P)=1$, while Proposition 12 says that $\alpha_{\varphi}(P) \leq \delta_{\varphi}$. The arithmetic degree can thus be used to stratify the points in $\mathbb{P}^{N}(\overline{\mathbb{Q}})$. Conjecture $1\left(\right.$ a) says that $\alpha_{\varphi}(P)$ takes on only finitely many values. What do sets of the form

$$
\left\{Q \in \mathbb{P}^{N}(\overline{\mathbb{Q}})_{\varphi}: \alpha_{\varphi}(Q)=\alpha_{\varphi}(P)\right\}
$$

look like for the finitely many possible values of $\alpha_{\varphi}(P)$ ?

\section{CANONiCAL heights FOR DOMinant RATional MAPS}

In this section we define and study basic properties of canonical heights for general dominant rational maps. Later we give refined results for monomial maps.

Definition. Let $\varphi: \mathbb{P}^{N} \rightarrow \mathbb{P}^{N}$ be a dominant rational map defined over $\overline{\mathbb{Q}}$ with dynamical degree $\delta_{\varphi}$ and associated quantity $\ell_{\varphi}$ as defined in Conjecture 2. Assume that $\delta_{\varphi}>1$. Let $P \in \mathbb{P}^{N}(\overline{\mathbb{Q}})_{\varphi}$. The canonical height of $P$ (relative to $\varphi$ ) is the quantity

$$
\hat{h}_{\varphi}(P)=\limsup _{n \rightarrow \infty} \frac{1}{n^{\ell} \delta_{\varphi}^{n}} h\left(\varphi^{n}(P)\right)
$$

We give an example to show that the limsup is necessary in the definition of the canonical height. 
Example 14. Let $d \geq 2$ be an integer, and let $\varphi: \mathbb{P}^{2} \rightarrow-\mathbb{P}^{2}$ be the map $\varphi(x, y)=\left(x^{-d}, y^{-d}\right)$. In homogeneous coordinates, we have

$$
\varphi^{n}([X, Y, 1])= \begin{cases}{\left[X^{d^{n}}, Y^{d^{n}}, 1\right]} & \text { if } n \text { is even, } \\ {\left[Y^{d^{n}}, X^{d^{n}}, X^{d^{n}} Y^{d^{n}}\right]} & \text { if } n \text { is odd. }\end{cases}
$$

Thus $\operatorname{deg}\left(\varphi^{n}\right)=d^{n}$ if $n$ is even, and $\operatorname{deg}\left(\varphi^{n}\right)=2 d^{n}$ if $n$ is odd, so in particular $\delta_{\varphi}=d$ and $\ell_{\varphi}=0$.

We now consider points $P=(x, y) \in \mathbb{Z}^{2}$ with $x y \neq 0$. Then

$$
d^{-n} h\left(\varphi^{n}(P)\right)= \begin{cases}\log \max \{|x|,|y|\} & \text { if } n \text { is even, } \\ \log (|x y|) & \text { if } n \text { is odd. }\end{cases}
$$

Thus the sequence $\delta_{\varphi}^{-n} h\left(\varphi^{n}(P)\right)$ does not have a limit (unless $|x|=1$ or $|y|=1)$.

Question 15. Is it true that the sequence $n^{-\ell_{\varphi}} \delta_{\varphi}^{-n} h\left(\varphi^{n}(P)\right)$ has only finitely many accumulation points in $\mathbb{R} \cup\{\infty\}$ ?

Our next example shows that the $n^{\ell}$ factor in the definition of $\hat{h}_{\varphi}$ is necessary if we want the canonical height to be finite.

Example 16. Consider the map $\varphi([1, x, y])=\left[1, x^{d} y, y^{d}\right]$ with $d \geq 2$. Then

So

$$
\varphi^{n}([1, x, y])=\left[1, x^{d^{n}} y^{n d^{n-1}}, y^{d^{n}}\right]
$$

$$
\delta_{\varphi}=\lim _{n \rightarrow \infty}\left(d^{n}+n d^{n-1}\right)^{1 / n}=d \quad \text { and } \quad \ell_{\varphi}=1 .
$$

Then for integers $x$ and $y$ with $x y \neq 0$, we have

$$
\begin{aligned}
\hat{h}_{\varphi}([1, x, y]) & =\limsup _{n \rightarrow \infty} n^{-1} d^{-n} h\left(\left[1, x^{d^{n}} y^{n d^{n-1}}, y^{d^{n}}\right]\right) \\
& =\limsup _{n \rightarrow \infty} n^{-1} d^{-n} \log \left|x^{d^{n}} y^{n d^{n-1}}\right| \\
& =\limsup _{n \rightarrow \infty}\left(\frac{1}{n} \log |x|+\frac{1}{d} \log |y|\right)=\frac{1}{d} \log |y| .
\end{aligned}
$$

Unfortunately, as the next example shows, the assumption that $\ell_{\varphi}>$ 0 does not suffice to imply that $\hat{h}_{\varphi}(P)$ is finite.

Example 17. Let $\varphi: \mathbb{P}^{3} \rightarrow \mathbb{P}^{3}$ be the map given in affine coordinates by

$$
\varphi(x, y, z)=(x y+x z, y+z, z)
$$

Then

$$
\varphi^{n}(x, y, z)=(x(y+z)(y+2 z) \cdots(y+n z), y+n z, z),
$$


SO

$$
\operatorname{deg}\left(\varphi^{n}\right)=n+1, \quad \delta_{\varphi}=1, \quad \text { and } \quad \ell_{\varphi}=1 .
$$

On the other hand, we have

$$
\frac{h\left(\varphi^{n}(1,0,1)\right)}{n^{\ell} \delta_{\varphi}^{n}}=\frac{h(n !, n, 1)}{n}=\frac{\log (n !)}{n} \sim \log n \quad \text { as } n \rightarrow \infty .
$$

Hence for this example we have $\hat{h}_{\varphi}(1,0,1)=\infty$.

Question 18. If $\delta_{\varphi}>1$, is it true that the canonical height $\hat{h}_{\varphi}(P)$ is finite? Example 17 shows that the answer is negative if $\delta_{\varphi}=1$, even if we require that $\ell_{\varphi}>0$.

In Section 6 we prove that Question 18 has an affirmative answer for monomial maps; see Proposition 25.

Proposition 19. The canonical height has the following properties:

(a) $0 \leq \hat{h}_{\varphi}(P) \leq \infty$.

(b) $\hat{h}_{\varphi}(\varphi(P))=\delta_{\varphi} \hat{h}_{\varphi}(P)$.

(c) If $P \in \operatorname{PrePer}(\varphi)$, then $\hat{h}_{\varphi}(P)=0$.

(d) If $\hat{h}_{\varphi}(P)>0$, then $\alpha_{\varphi}(P)=\delta_{\varphi}$.

Proof. (a) This is obvious, since the height $h$ is a non-negative function. (b) We compute

$$
\begin{aligned}
\hat{h}_{\varphi}\left(\varphi^{n}(P)\right) & =\limsup _{n \rightarrow \infty} \frac{1}{n^{\ell} \delta_{\varphi}^{n}} h\left(\varphi^{n+1}(P)\right) \\
& =\limsup _{n \rightarrow \infty} \frac{1}{(n-1)^{\ell_{\varphi}} \delta_{\varphi}^{n-1}} h\left(\varphi^{n}(P)\right) \\
& =\delta_{\varphi} \limsup _{n \rightarrow \infty}\left(\frac{n}{n-1}\right)^{\ell_{\varphi}} \frac{1}{n^{\ell_{\varphi} \delta_{\varphi}^{n}}} h\left(\varphi^{n}(P)\right) \\
& =\delta_{\varphi} \hat{h}_{\varphi}(P) .
\end{aligned}
$$

(c) If $P$ is preperiodic, then $h\left(\varphi^{n}(P)\right)$ takes on only finitely many values, so it is immediate from the defintion of $\hat{h}_{\varphi}$ that $\hat{h}_{\varphi}(P)=0$.

(d) We are assuming that $\hat{h}_{\varphi}(P)>0$, and by definition $\hat{h}_{\varphi}(P)$ is the limsup of $n^{-\ell \varphi} \delta_{\varphi}^{-n} h\left(\varphi^{n}(P)\right)$, so we can find an infinite sequence $\mathcal{N}$ of positive integers such that

$$
n^{-\ell_{\varphi}} \delta_{\varphi}^{-n} h\left(\varphi^{n}(P)\right) \geq \frac{1}{2} \hat{h}_{\varphi}(P)>0 \quad \text { for all } n \in \mathcal{N} .
$$

It follows that

$$
\alpha_{\varphi}(P)=\limsup _{n \rightarrow \infty} h\left(\varphi^{n}(P)\right)^{1 / n} \geq \limsup _{n \in \mathcal{N}}\left(n^{\ell_{\varphi}} \delta_{\varphi}^{n} \cdot \frac{1}{2} \hat{h}_{\varphi}(P)\right)^{1 / n}=\delta_{\varphi}
$$


But we know from Proposition 12 that $\alpha_{\varphi}(P) \leq \delta_{\varphi}$ for every dominant rational map $\varphi$, so this proves that $\alpha_{\varphi}(P)=\delta_{\varphi}$.

Remark 20. The implication

$$
P \in \operatorname{PrePer}(\varphi) \quad \Longrightarrow \quad \hat{h}_{\varphi}(P)=0
$$

in Proposition 19(c) is trivial, but for applications one generally wants to know that the opposite implication holds, at least off of an explicitly described exceptional set. One way to prove the opposite implication is to show that $\hat{h}_{\varphi}(P)$ is equal to $h(P)+O(1)$, or at least satisfies $\hat{h}_{\varphi}(P) \asymp h(P)$, again off of an exceptional set. ${ }^{1}$ From such an estimate, it immediately follows that

$$
\begin{aligned}
\hat{h}_{\varphi}(P)=0 & \Longrightarrow 0=\delta_{\varphi}^{n} \hat{h}_{\varphi}(P)=\hat{h}_{\varphi}\left(\varphi^{n}(P)\right) \\
& \Longrightarrow h\left(\varphi^{n}(P)\right) \asymp 0 \\
& \Longrightarrow \mathcal{O}_{\varphi}(P) \text { is a set of bounded height, } \\
& \Longrightarrow \mathcal{O}_{\varphi}(P) \text { is a finite set. }
\end{aligned}
$$

When $\varphi$ is a birational map, another method used to prove the reverse implication is to use an estimate of the form

$$
C_{1} h(\varphi(P))+C_{2} h\left(\varphi^{-1}(P)\right) \geq C_{3} h(P)+O(1) ;
$$

see for example $[20,38,39,42,46,55,56]$ for results of this type for regular affine automorphisms.

Corollary 31 says that the reverse implication

$$
\hat{h}_{\varphi}(P)=0 \quad \Longrightarrow \quad P \in \operatorname{PrePer}(\varphi)
$$

is true for a certain (large) class of monomial maps on $\mathbb{P}^{N}$, but the proof is not via an estimate $\hat{h}_{\varphi}(P) \asymp h(P)$.

\section{MONOMIAL MAPS AND CANONICAL HEIGHTS}

A monomial map is an endomorphism of the torus $\mathbb{G}_{m}^{N}$. Embedding $\mathbb{G}_{m}^{N}$ in $\mathbb{P}^{N}$, monomial maps induce rational self-maps of $\mathbb{P}^{N}$. In this section we study the geometry of iteration of these maps and prove that the canonical height is finite. We begin with a formal definition which sets the notation that we will use throughout the rest of this article.

\footnotetext{
${ }^{1}$ For nonnegative functions $F$ and $G$, we write $F \asymp G$ to mean that there are positive constants $c_{1}, c_{2}, c_{3}, c_{4}$ such that $c_{1} F(x)-c_{2} \leq G(x) \leq c_{3} F(x)+c_{4}$.
} 
Definition. We write $\operatorname{Mat}_{N}^{+}(\mathbb{Z})$ for the set of $N$-by- $N$ matrices with integer coefficients and nonzero determinant. To each matrix $A \in$ $\operatorname{Mat}_{N}^{+}(\mathbb{Z})$ we associate the monomial map $\varphi_{A}: \mathbb{G}_{m}^{N} \rightarrow \mathbb{G}_{m}^{N}$ given by the formula

$$
\begin{aligned}
& \varphi_{A}\left(X_{1}, \ldots, X_{N}\right)= \\
& \quad\left(X_{1}^{a_{11}} X_{2}^{a_{12}} \cdots X_{N}^{a_{1 N}}, X_{1}^{a_{21}} X_{2}^{a_{22}} \cdots X_{N}^{a_{2 N}}, \ldots, X_{1}^{a_{N 1}} X_{2}^{a_{N 2}} \cdots X_{N}^{a_{N N}}\right) .
\end{aligned}
$$

We call $\varphi_{A}$ the monomial map associated to $A$. We note that $\varphi_{A}$ induces a rational map $\varphi_{A}: \mathbb{P}^{N} \rightarrow \mathbb{P}^{N}$. We denote the spectral radius of $A$ by

$$
\rho(A)=\max \{|\lambda|: \lambda \in \mathbb{C} \text { is an eigenvalue for } A\} .
$$

It is immediate from the definition that if $A, B \in \operatorname{Mat}_{N}^{+}(\mathbb{Z})$ are matrices with associated monomial maps $\varphi_{A}$ and $\varphi_{B}$, then

$$
\varphi_{A B}(P)=\left(\varphi_{A} \circ \varphi_{B}\right)(P) \text { and } \varphi_{A+B}(P)=\varphi_{A}(P) \cdot \varphi_{B}(P) .
$$

Proposition 21. Let $A \in \operatorname{Mat}_{N}^{+}(\mathbb{Z})$ be a matrix with associated monomial map $\varphi_{A}$.

(a) $\rho(A) \geq 1$.

(b) $\rho(A)=1$ if and only if all of the eigenvalues of $A$ are roots of unity, which is equivalent to $\left(A^{m}-I\right)^{n}=0$ for some positive integers $m$ and $n$.

(c) (Hasselblatt-Propp [33]; see also [43]) The dynamical degree of $\varphi_{A}$ is equal to its spectral radius,

$$
\delta_{\varphi_{A}}=\rho(A) .
$$

(d) If none of the eigenvalues of $A$ are roots of unity, then the set of preperiodic points of $\varphi_{A}$ in $\mathbb{G}_{m}^{N}(\mathbb{C})$ is

$$
\operatorname{PrePer}\left(\varphi_{A}\right)=\mathbb{G}_{m}^{N}(\overline{\mathbb{Q}})_{\text {tors }},
$$

where the torsion subgroup $\mathbb{G}_{m}^{N}(\overline{\mathbb{Q}})_{\text {tors }}$ is the set of points whose coordinates are roots of unity.

Proof. Let $\lambda_{1}, \ldots, \lambda_{N}$ be the eigenvalues of $A$, labeled so that $\left|\lambda_{1}\right|=$ $\rho(A)$. The product $\lambda_{1} \lambda_{2} \cdots \lambda_{N}$ of the eigenvalues equals $\operatorname{det}(A)$, which is a non-zero integer, so certainly $\left|\lambda_{1}\right| \geq 1$. This proves (a)

If $\rho(A)=1$, then for every $i$, the algebraic integer $\lambda_{i}$ has the property that all of its Galois conjugates are in the closed unit circle. It follows from Kronecker's theorem [57, Theorem 3.8] that $\lambda_{i}$ is a root of unity. Thus all of the eigenvalues of $A$ are roots of unity, so $A$ is quasi-unipotent. Conversely, if $A$ is quasi-unipotent, then its characteristic polynomial divides $\left(T^{n}-1\right)^{m}$ for some $n \geq 1$ and $m \geq 1$, so the eigenvalues of $A$ are roots of unity, hence have absolute value equal to 1 . This proves the first part of (b), and the second part is easy. 
The fact that $\delta_{\varphi_{A}}=\rho(A)$ is due to Hasselblatt and Propp [33, Theorem 6.2], which gives (c).

Finally, for (d), we use (9) to see that

$$
\varphi_{A}^{n}(P)=\varphi_{A}^{m}(P) \Longleftrightarrow \varphi_{A^{n}}(P)=\varphi_{A^{m}}(P) \Longleftrightarrow \varphi_{A^{n}-A^{m}}(P)=1 .
$$

Thus (b) and the following lemma complete the proof of $(d)$.

Lemma 22. Let $B \in \operatorname{Mat}_{N}^{+}(\mathbb{Z})$ be a matrix with $\operatorname{det}(B) \neq 0$, and suppose that a point $P \in \mathbb{G}_{m}^{N}(\mathbb{C})$ satisfies

$$
\varphi_{B}(P)=1 \text {. }
$$

Then every coordinate of $P$ is a root of unity.

Proof. For notational clarity, we write $\mathbf{e}=(1,1, \ldots, 1)$ for the identity element of $\mathbb{G}_{m}^{N}(\mathbb{C})$, so our assumption is that $\varphi_{B}(P)=$ e. Let $\Delta=$ $\operatorname{det}(B)$, and let $C=B^{\text {adj }}$ be the adjoint matrix, so $C B=\Delta I_{N}$. Then

$$
\mathbf{e}=\varphi_{C}(\mathbf{e})=\varphi_{C} \circ \varphi_{B}(P)=\varphi_{C B}(P)=\varphi_{\Delta I_{N}}(P) .
$$

Hence every coordinate of $P$ is a $\Delta^{\text {th }}$-root of unity. [I thank Jan-Li Lin (private communication) for showing me this proof. More generally, for any $B \in \operatorname{Mat}_{N}(\mathbb{Z})$, the map $\varphi_{B}$ is an endomorphism of $\mathbb{G}_{m}^{N}$, so its kernel is an algebraic subgroup with codimension equal to the rank of $B$.]

Remark 23. The restriction in Proposition 21(d) that $A$ has no eigenvalues that are roots of unity is necessary, as is seen for example from the map

$$
\varphi(x, y)=\left(x^{a} y^{1-a}, x^{b} y^{1-b}\right) \quad \text { associated to the matrix } \quad A=\left(\begin{array}{cc}
a & 1-a \\
b & 1-b
\end{array}\right) .
$$

Then 1 is an eigenvalue of $A$, and $\varphi(t, t)=(t, t)$, so $(t, t)$ is a fixed point for every $t$.

Lin, Jonsson, and Wulcan have proven a strengthened version of Conjecture 2 for monomial maps.

Theorem 24. (Lin [44, Theorem 6.2], Jonsson-Wulcan [36]) Let $A \in$ $\operatorname{Mat}_{N}^{+}(\mathbb{Z})$, let $\varphi_{A}$ be the associated monomial map, and let $\ell(A)+1$ be the dimension of the largest Jordan block of $A$ among those blocks corresponding to eigenvalues of maximal absolute value. Then

$$
\operatorname{deg}\left(\varphi_{A}^{n}\right) \asymp n^{\ell(A)} \rho(A)^{n} \quad \text { for all } n \geq 1,
$$

where the implied constants depend only on $A$. In particular, $\ell(A)$ is an integer satisfying $0 \leq \ell(A)<N$. 
Lin proves Theorem 24 by extending the degree map $A \mapsto \operatorname{deg}\left(\varphi_{A}\right)$ to a function on $\mathrm{Mat}_{N}^{+}(\mathbb{R})$, showing that the resulting function is moreor-less a norm, and using a compactness argument. We can use Theorem 24 and an elementary argument to prove that the canonical height for monomial maps is finite.

Proposition 25. Let $\varphi_{A}$ be a monomial map associated to a matrix $A \in \operatorname{Mat}_{N}^{+}(\mathbb{Z})$ with either $\rho(A)>0$ or $\ell(A)>0$, where $\rho(A)$ is the spectral radius of $A$ and $\ell(A)$ is as in the statement of Theorem 24. Then there is a constant $C(A)$ such that

$$
\hat{h}_{\varphi_{A}}(P) \leq C(A) h(P) \quad \text { for all } P \in \mathbb{G}_{m}^{N}(\overline{\mathbb{Q}}) .
$$

In particular, the canonical height $\hat{h}_{\varphi_{A}}(P)$ is finite.

Proof. Theorem 24 and the definition of dynamical degree imply that $\delta_{\varphi}=\rho(A)$ and $\ell_{\varphi}=\ell(A)$, so the definition of the canonical height becomes

$$
\hat{h}_{\varphi_{A}}(P)=\limsup _{n \rightarrow \infty} \frac{1}{n^{\ell(A)} \rho(A)^{n}} h\left(\varphi^{n}(P)\right) .
$$

For a matrix $C=\left(c_{i j}\right) \in \operatorname{Mat}_{N}(\mathbb{C})$, we write $\|C\|_{\infty}$ for the supnorm $\max \left|c_{i j}\right|$. An elementary triangle inequality estimate (cf. [44, Lemma 6.4]) gives

$$
\left\|A^{n}\right\|_{\infty} \ll n^{\ell(A)} \rho(A)^{n} .
$$

(In fact, $\left\|A^{n}\right\|_{\infty} \asymp n^{\ell(A)} \rho(A)^{n}$.)

We write

and we let

$$
A^{n}=\left(a_{i j}(n)\right)_{1 \leq i, j \leq N},
$$

$$
P=\left(x_{1}, \ldots, x_{N}\right) \text { and } \varphi_{A}^{n}(P)=\left(y_{1}, \ldots, y_{N}\right) .
$$

Then

$$
\begin{aligned}
h\left(\varphi^{n}(P)\right) & =\sum_{v \in M_{K}} \max _{1 \leq i \leq N} \log ^{+}\left\|y_{i}\right\|_{v} \\
& =\sum_{v \in M_{K}} \max _{1 \leq i \leq N}\left\{0, \sum_{j=1}^{N} a_{i j}(n) \log \left\|x_{i}\right\|_{v}\right\} \\
& \leq N\left\|A^{n}\right\|_{\infty} \sum_{v \in M_{K}} \max _{1 \leq i \leq N} \log ^{+}\left\|x_{i}\right\|_{v} \\
& \ll n^{\ell(A)} \rho(A)^{n} h(P) \quad \text { from }(12),
\end{aligned}
$$

where the implied constant depends on $N$ and $A$, but is independent of $n$ and $P$. This inequality shows that the limsup in (11) is finite and bounded by a constant multiple of $h(P)$. 
Remark 26. We note that

$$
\hat{h}_{\varphi_{A}}\left(x_{1}, \ldots, x_{N}\right)=\hat{h}_{\varphi_{A}}\left(\zeta_{1} x_{1}, \ldots, \zeta_{N} x_{N}\right)
$$

for any roots of unity $\zeta_{1}, \ldots, \zeta_{N}$. In particular, the set of points satisfying $\hat{h}_{\varphi_{A}}(P)=0$ is invariant under multiplying the coordinates of the points by roots of unity.

\section{POINTS OF CANONICAL HEIGHT ZERO FOR MONOMIAL MAPS}

It is a trivial fact (Proposition 19(c)) that preperiodic points have canonical height zero. The converse is not true in full generality, and it can be quite delicate to determine the set of points having canonical height zero. In this section we prove, among other things, that the converse is true for monomial maps whose associated matrix has irreducible characteristic polynomial. We start with a general result which says that for monomial maps, the set of points of canonical height zero lies in a proper algebraic subgroup.

Definition. Let $A \in \mathrm{GL}_{N}(\mathbb{Q})$. A Jordan subspace for $A$ is an $A$ invariant subspace of $\overline{\mathbb{Q}}^{N}$ corresponding to a single Jordan block of $A$. A Jordan subspace $V \subset \overline{\mathbb{Q}}^{N}$ with associated eigenvalue $\lambda$ is called a maximal Jordan subspace if $|\lambda|=\rho(A)$ and if the dimension of $V$ is maximal among the Jordan subspaces whose eigenvalue have magnitude equal to $\rho(A)$. We set

$$
\begin{aligned}
& r(A)=\text { number of maximal Jordan subspaces, } \\
& \bar{r}(A)=\#\left\{\sigma(V): \begin{array}{l}
V \text { is a maximal Jordan subspace } \\
\text { for } A \text { and } \sigma \in \operatorname{Gal}(\overline{\mathbb{Q}} / \mathbb{Q})
\end{array}\right\} .
\end{aligned}
$$

Thus $\bar{r}(A)$ is the number of distinct $\overline{\mathbb{Q}}$-subspaces of $\overline{\mathbb{Q}}^{N}$ that are Galois conjugate to a maximal Jordan subspace of $A$. We note that $\bar{r}(A) \geq$ $r(A) \geq 1$, since $A$ always has at least one maximal Jordan subspace.

Definition. Let $G$ be an algebraic subgroup of $\mathbb{G}_{m}^{N}$. We write $G(\overline{\mathbb{Q}})^{\text {div }}$ for the divisible hull of $G(\overline{\mathbb{Q}})$,

$$
\begin{aligned}
G(\overline{\mathbb{Q}})^{\operatorname{div}}=\left\{\left(\alpha_{1}, \ldots, \alpha_{N}\right)\right. & \in \mathbb{G}_{m}^{N}(\overline{\mathbb{Q}}) \\
& \left.:\left(\alpha_{1}^{n}, \ldots, \alpha_{N}^{n}\right) \in G(\overline{\mathbb{Q}}) \text { for some } n \geq 1\right\} .
\end{aligned}
$$

Equivalently, $G(\overline{\mathbb{Q}})^{\text {div }}$ is the set of translates of $G(\overline{\mathbb{Q}})$ by points in $\mathbb{G}_{m}^{N}(\overline{\mathbb{Q}})_{\text {tors }}{ }^{2}$

We can now state our main result.

\footnotetext{
${ }^{2}$ For further details, see Appendix A.1.
} 
Theorem 27. Let $A \in \operatorname{Mat}_{N}^{+}(\mathbb{Z})$ be a matrix whose associated monomial map $\varphi_{A}$ has dynamical degree $\delta_{\varphi_{A}}>1$. There is an algebraic subgroup $G \subset \mathbb{G}_{m}^{N}$ with dimension

$$
\operatorname{dim} G \geq N-\bar{r}(A)
$$

such that

$$
\left\{P \in \mathbb{G}_{m}^{N}(\overline{\mathbb{Q}}): \hat{h}_{\varphi_{A}}(P)=0\right\} \subset G(\overline{\mathbb{Q}})^{\text {div }} .
$$

Remark 28. The proof of Theorem 27 describes explicitly how to construct the group $G$ from the matrix $A$.

Theorem 27 has a number of interesting corollaries.

Corollary 29. Let $\varphi: \mathbb{G}_{m}^{N} \rightarrow \mathbb{G}_{m}^{N}$ be a monomial map with $\delta_{\varphi}>1$, and let $P \in \mathbb{G}_{m}^{N}(\overline{\mathbb{Q}})$ be a point with $\hat{h}_{\varphi}(P)=0$. Then there is a proper algebraic subgroup $G \subsetneq \mathbb{G}_{m}^{N}$ with $\mathcal{O}_{\varphi}(P) \subset G$. In particular, the orbit $\mathcal{O}_{\varphi}(P)$ is not Zariski dense in $\mathbb{G}_{m}^{N}$.

Remark 30. It should be possible to use an effective form of Baker's theorem to prove effective versions of Theorem 27 and Corollary 29. Thus for example, the proofs should yield an effective constant $C=$ $C(A, h(P))>0$ such that

$$
\mathcal{O}_{\varphi}(P) \text { Zarisiki dense } \Longrightarrow \hat{h}_{\varphi_{A}}(P)>C .
$$

Presumably the constant $C$ computed in this way is very small if the coefficients of $A$ or the height of $P$ is large. It is an interesting question as to whether a Lehmer-type estimate holds, e.g., is it possible to take $C=C^{\prime} h(P)^{-k}$ for constants $C^{\prime}$ and $k$ that depend only on $A$ ? (Maybe even with $k$ depending only on $N$ ?)

For monomial maps whose associated matrices have irreducible characteristic polynomial, we can say more.

Corollary 31. Let $\varphi: \mathbb{G}_{m}^{N} \rightarrow \mathbb{G}_{m}^{N}$ be a monomial map with dynamical degree $\delta_{\varphi}>1$ defined by a matrix $A \in \operatorname{Mat}_{N}^{+}(\mathbb{Z})$ whose characteristic polynomial is irreducible over $\mathbb{Q}$. Let $P \in \mathbb{G}_{m}^{N}(\overline{\mathbb{Q}})$. Then

$$
\hat{h}_{\varphi}(P)=0 \Longleftrightarrow P \in \operatorname{PrePer}(\varphi) \text {. }
$$

We next describe the set of arithmetic degrees for a monomial map.

Definition. For a polynomial $f(T) \in \mathbb{C}[T]$, we write

$$
\rho(f)=\max \{|\alpha|: \alpha \in \mathbb{C} \text { is a root of } f\} .
$$

With this notation, the spectral radius of a matrix $A \in \operatorname{Mat}_{N}(\mathbb{C})$ is $\rho(A)=\rho(\operatorname{det}(T-A))$. 
Corollary 32. Let $\varphi: \mathbb{G}_{m}^{N} \rightarrow \mathbb{G}_{m}^{N}$ be a monomial map defined by a matrix $A \in \operatorname{Mat}_{N}^{+}(\mathbb{Z})$, and let $f_{1}(T), \ldots, f_{s}(T) \in \mathbb{Z}[T]$ be the monic irreducible factors of the characteristic polynomial $\operatorname{det}(T-A)$ of $A$. Then

$$
\left\{\alpha_{\varphi}(P): P \in \mathbb{G}_{m}^{N}(\overline{\mathbb{Q}})\right\}=\left\{1, \rho\left(f_{1}\right), \rho\left(f_{2}\right), \ldots, \rho\left(f_{s}\right)\right\} .
$$

In particular, for every $P \in \mathbb{G}_{m}^{N}(\overline{\mathbb{Q}})$ the algebraic degree $\alpha_{\varphi}(P)$ is an algebraic integer, and $\alpha_{\varphi}(P)$ takes on only finitely many values as $P$ ranges over $\mathbb{G}_{m}^{N}(\overline{\mathbb{Q}})$.

All rational maps have the property that if $\hat{h}_{\varphi}(P)>0$, then $\alpha_{\varphi}(P)=$ $\delta_{\varphi}$; see Proposition $19(\mathrm{~d})$. For monomial maps whose associated matrices are diagonalizable, we can prove the converse. We note that some restriction is necessary, since it is easy to construct non-diagonalizable monomial maps for which Corollary 33 is false. Indeed, the map in Example 16 applied to the point $P=[1,2,1]$ provides an example with $\hat{h}_{\varphi}(P)=0$ and $\alpha_{\varphi}(P)=\delta_{\varphi}$. (I thank Mattias Jonsson for this last observation.)

Corollary 33. Let $A \in \operatorname{Mat}_{N}^{+}(\mathbb{Z})$ be a matrix that is diagonalizable over $\mathbb{C}$, and assume that the associated monomial map $\varphi: \mathbb{P}^{N} \rightarrow \mathbb{P}^{N}$ satisfies $\delta_{\varphi}>1$. Let $P \in \mathbb{G}_{m}^{N}(\overline{\mathbb{Q}})$. Then

$$
\hat{h}_{\varphi}(P)>0 \Longleftrightarrow \alpha_{\varphi}(P)=\delta_{\varphi} \text {. }
$$

\section{Proof of Theorem 27}

In this section we give the proof of Theorem 27. We start with some additional notation.

Definition. By definition, all of the maximal Jordan subspaces for a matrix $A$ have the same dimension. We let

$$
\ell(A)=\operatorname{dim}(\text { any maximal Jordan subspace })-1 \text {. }
$$

We remark that if $\varphi_{A}$ is the monomial map associated to a matrix $A \in$ $\operatorname{Mat}_{N}^{+}(\mathbb{Z})$ and $\ell_{\varphi_{A}}$ is the associated degree growth exponent defined in Conjecture 2, then Proposition 24 implies that $\ell(A)=\ell_{\varphi_{A}}$, so our use of $\ell$ for two seemingly different purposes is consistant.

Definition. Let $K / F$ be an extension of fields, and let $W \subset K^{N}$ be a set of vectors. We write

$$
\operatorname{Perp}_{F}(W)=\left\{\mathbf{b} \in F^{N}: \mathbf{b} \cdot \mathbf{w}=0 \text { for all } \mathbf{w} \in W\right\}
$$

for the subspace of $F^{N}$ that is orthogonal to $W$. When $W=\{\mathbf{w}\}$ consists of a single vector, we write

$$
\operatorname{Perp}_{F}(\mathbf{w})=\left\{\mathbf{b} \in F^{N}: \mathbf{b} \cdot \mathbf{w}=0\right\}
$$


for $\operatorname{Perp}_{F}(\{\mathbf{w}\})$. Assuming that $\mathbf{w} \neq \mathbf{0}$, we note that $\operatorname{Perp}_{K}(\mathbf{w})$ is simply a hyperplane in $K^{N}$, but that in general the $F$-vector space $\operatorname{Perp}_{F}(\mathbf{w})$ may have dimension anywhere from 0 to $N-1$.

The following elementary facts will be useful.

Lemma 34. Let $K / F$ be an extension of fields.

(a) Let $U \subset F^{N}$ be an F-vector subspace. Then

$$
\operatorname{Perp}_{K}\left(U \otimes_{F} K\right)=\operatorname{Perp}_{F}(U) \otimes_{F} K \text {. }
$$

(b) Assume that $K / F$ is Galois, and let $V \subset K^{N}$ be a $K$-vector subspace that is $\operatorname{Gal}(K / F)$-invariant. Then $Y=V \cap F^{N}$ is the unique $F$-vector subspace of $F^{N}$ satisfying $V=Y \otimes_{F} K$.

(c) Let $V \subset F^{N}$ be an F-vector subspace. Then

$$
\operatorname{Perp}_{F}\left(\operatorname{Perp}_{F}(V)\right)=V \text {. }
$$

(d) Let $V_{1}, \ldots, V_{t} \subset F^{N}$ be $F$-vector subspaces. Then

$$
\operatorname{Perp}_{F}\left(V_{1}\right)+\cdots+\operatorname{Perp}_{F}\left(V_{t}\right)=\operatorname{Perp}_{F}\left(V_{1} \cap \cdots \cap V_{t}\right) .
$$

Proof. (a), (c), and (d) are linear algebra exercises, while (b) is standard linear algebra and Galois theory; cf. [58, Lemma II.5.8.1]. ${ }^{3}$

The proof of Theorem 27 uses the following (qualitative) version of Baker's theorem on linear forms in logarithms.

Theorem 35. (Baker's Theorem) Let $\alpha_{1}, \ldots, \alpha_{n} \in \overline{\mathbb{Q}}^{*}$ be algebraic numbers, and let

$$
\mathbf{w}=\left(\log \left(\alpha_{1}\right), \ldots, \log \left(\alpha_{n}\right)\right) \in \mathbb{C}^{n}
$$

Then

$$
\operatorname{Perp}_{\overline{\mathbb{Q}}}(\mathbf{w}) \cong \operatorname{Perp}_{\mathbb{Q}}(\mathbf{w}) \otimes_{\mathbb{Q}} \overline{\mathbb{Q}} \text {. }
$$

In particular, the $\overline{\mathbb{Q}}$-vector space $\operatorname{Perp}_{\overline{\mathbb{Q}}}(\mathbf{w}) \subset \overline{\mathbb{Q}}^{N}$ is $\operatorname{Gal}(\overline{\mathbb{Q}} / \mathbb{Q})$-invariant.

Proof. If $\operatorname{dim} \operatorname{Perp}_{\overline{\mathbb{Q}}}(\mathbf{w})=0$ or 1 , then (13) says that

$$
\log \left(\alpha_{1}\right), \ldots, \log \left(\alpha_{n}\right)
$$

are linearly dependent over $\overline{\mathbb{Q}}$ if and only if they are linearly dependent over $\mathbb{Q}$, which is the usual statement of Baker's theorem; see [6]. The general case of (13) is then an easy induction on the dimension of $\operatorname{Perp}_{\overline{\mathbb{Q}}}(\mathbf{w}){ }^{4}$ Finally, the equality (13) shows that $\operatorname{Perp}_{\overline{\mathbb{Q}}}(\mathbf{w})$ is a $\operatorname{Gal}(\overline{\mathbb{Q}} / \mathbb{Q})$-invariant subspace of $\overline{\mathbb{Q}}^{N}$.

\footnotetext{
${ }^{3}$ For further details, see Appendix A.2.

${ }^{4}$ For further details, see Appendix A.3.
} 
Proof of Theorem 27. To ease notation, we let $\rho=\rho(A)$ and $\ell=$ $\ell(A)=\ell_{\varphi}$. We also note from Proposition 21(c) that $\rho=\delta_{\varphi_{A}}$, so $\rho>1$. We take $K / \mathbb{Q}$ to be a finite Galois extension containing the coordinates of $P$ and the eigenvalues of $A$.

Writing $A$ in Jordan normal form, it is easy to see that the matrices

$$
\left\{n^{-\ell} \rho^{-n} A^{n}: n \geq 0\right\}
$$

lie in a bounded subset of $\operatorname{Mat}_{N}(\mathbb{C}) \cong \mathbb{C}^{N^{2}}$, so by compactness, any infinite subsequence of matrices in (14) has an accumulation point in $\operatorname{Mat}_{N}(\mathbb{C})$.

For $Q=\left(y_{1}, \ldots, y_{N}\right) \in K^{N}$ and $v \in M_{K}$, we let

$$
\log \|Q\|_{v}=\text { the column vector }{ }^{t}\left(\log \left\|y_{1}\right\|_{v}, \ldots, \log \left\|y_{N}\right\|_{v}\right) .
$$

This notation gives the convenient formula

$$
\log \left\|\varphi_{A}^{n}(P)\right\|_{v}=A^{n} \log \|P\|_{v}
$$

Further, for any real vector $\mathbf{u}=\left(u_{1}, \ldots, u_{N}\right) \in \mathbb{R}^{N}$, we let

$$
\max (\mathbf{u})=\max \left\{u_{1}, \ldots, u_{N}\right\} \quad \text { and } \max ^{+}(\mathbf{u})=\max \left\{0, u_{1}, \ldots, u_{N}\right\} .
$$

Let $P \in \mathbb{G}_{m}^{N}(\overline{\mathbb{Q}})$ be a point satisfying $\hat{h}_{\varphi}(P)=0$, and let $v \in M_{K}$. Our first goal is to show that $\operatorname{Perp}_{\mathbb{C}}\left(\log \|P\|_{v}\right)$ contains a non-trivial $\mathbb{C}$-vector subspace of $\mathbb{C}^{N}$ that does not depend on $P$ or $v$. Using the definition of canonical height, we have

$$
0=\hat{h}_{\varphi}(P)=\limsup _{n \rightarrow \infty} \frac{h\left(\varphi_{A}^{n}(P)\right)}{n^{\ell} \rho^{n}} .
$$

We choose an infinite sequence of natural numbers $\mathcal{N}$ so that the limsup is a limit, i.e.,

$$
0=\hat{h}_{\varphi}(P)=\lim _{n \in \mathcal{N}} \frac{h\left(\varphi_{A}^{n}(P)\right)}{n^{\ell} \rho^{n}} .
$$

Replacing $\mathcal{N}$ with an infinite subsequence, which we again denote by $\mathcal{N}$, we may assume that the limit

$$
B=\lim _{n \in \mathcal{N}} \frac{A^{n}}{n^{\ell} \rho^{n}}
$$

also exists. We also note that since $A$ has integer coefficients, the coefficients of the matrix $B$ are real, i.e., $B \in \operatorname{Mat}_{N}(\mathbb{R})$. This is important because we are about to write down inequalities that involve the coefficients of $B$. On the other hand, it need not be true that the coefficients of $B$ are algebraic numbers, nor is $B$ necessarily invertible. 
Writing the height as a sum over the places of $K$, we have

$$
\begin{aligned}
0 & =\lim _{n \in \mathcal{N}} \frac{1}{n^{\ell} \rho^{n}} \sum_{v \in M_{K}} \max ^{+}\left(\log \left\|\varphi_{A}^{n}(P)\right\|_{v}\right) \quad \text { from (16), } \\
& =\lim _{n \in \mathcal{N}} \frac{1}{n^{\ell} \rho^{n}} \sum_{v \in M_{K}} \max ^{+}\left(A^{n} \log \|P\|_{v}\right) \quad \text { from (15), } \\
& =\sum_{v \in M_{K}} \max ^{+}\left(\lim _{n \in \mathcal{N}} \frac{A^{n}}{n^{\ell} \rho^{n}} \log \|P\|_{v}\right) \\
& =\sum_{v \in M_{K}} \max ^{+}\left(B \log \|P\|_{v}\right) \quad \text { from }(17) .
\end{aligned}
$$

Since this sum of non-negative terms is equal to 0 , we see that each individual term must be equal to 0 . We have thus proven that

$$
\max ^{+}\left(B \log \|P\|_{v}\right)=0 \quad \text { for all } v \in M_{K}
$$

where $B=\lim _{n \in \mathcal{N}} n^{-\ell} \rho^{-n} A^{n} \in \operatorname{Mat}_{N}(\mathbb{R})$ is given by (17).

The definition of $\max ^{+}$and (18) imply that ${ }^{5}$

$$
\max \left(B \log \|P\|_{v}\right) \leq 0 \quad \text { for all } v \in M_{K}
$$

However, summing over $v \in M_{K}$ and using the product formula shows that

$$
\sum_{v \in M_{K}} \max \left(B \log \|P\|_{v}\right) \geq 0
$$

Thus the sum (20) is non-negative, but (19) says that every term in the sum is non-positive. It follow that every term in the sum (20) must vanish, which proves the key formula

$$
B \log \|P\|_{v}=0 \quad \text { for all } v \in M_{K}
$$

We note that (21) is equivalent to

$$
\log \|P\|_{v} \in \operatorname{ker}_{\mathbb{C}}(B)
$$

where $\operatorname{ker}_{\mathbb{C}}(B) \subset \mathbb{C}^{N}$ is independent of both $P$ and $v$.

Formula (21) says that the row vectors of $B$ annihilate $\log \|P\|_{v}$, but unfortunately the coordinates of $B$ are in $\mathbb{C}$, and our ultimate goal is to find vectors with integer coordinates that annihilate $\log \|P\|_{v}$. To do this, we study $B$ and its kernel more closely.

Let $V \subset \overline{\mathbb{Q}}^{N}$ be a Jordan subspce for $A$, and let $t=\operatorname{dim} V$. In other words, $V$ is an $A$-invariant subspace of $\overline{\mathbb{Q}}^{N}$ and there is a $\overline{\mathbb{Q}}$-basis $\mathcal{V}$

\footnotetext{
${ }^{5}$ For further details, see Appendix A.4.
} 
for $V$ so that the matrix of $\left.A\right|_{V}$ relative to the basis $\mathcal{V}$ is

$$
\left[\left.A\right|_{V}\right]_{\mathcal{V}}=\left(\begin{array}{ccccc}
\lambda & 1 & 0 & \cdots & 0 \\
0 & \lambda & 1 & \cdots & 0 \\
\vdots & & \ddots & \ddots & \vdots \\
0 & 0 & & \cdots & \lambda
\end{array}\right)
$$

We consider the limiting action of $n^{-\ell} \rho^{-n} A^{n}$ on $V$. There are two cases.

First, if $V$ is not a maximal Jordan subspace, then either $|\lambda|<\rho$ or $t \leq \ell$, so

magnitude of largest entry of $\left[\left.A^{n}\right|_{V}\right]_{\mathcal{V}} \leq O\left(n^{t-1}|\lambda|^{n}\right)=o\left(n^{\ell} \rho^{n}\right)$.

Hence if $V$ is not a maximal Jordan subspace, then

$$
\left.\lim _{n \rightarrow \infty} n^{-\ell} \rho^{-n} A^{n}\right|_{V}=0
$$

Second, suppose that $V$ is a maximal Jordan subspace, so $|\lambda|=\rho$ and $t=\ell+1$. Let $\mathcal{V}=\left\{\mathbf{v}_{1}, \ldots, \mathbf{v}_{t}\right\}$ be the basis of $V$ used to put $\left[\left.A^{n}\right|_{V}\right]_{\mathcal{V}}$ into Jordan normal form, and let

$$
W=\overline{\mathbb{Q}} \mathbf{v}_{1}+\cdots+\overline{\mathbb{Q}} \mathbf{v}_{t-1} \subset V
$$

be the codimension 1 subspace of $V$ generated by the first $t-1$ vectors in the basis. Alternatively,

$$
W=\operatorname{ker}\left(\left.(A-\lambda)^{t-1}\right|_{V}\right) \subset V .
$$

Then $W$ is $A$-invariant, and the magnitude of the largest entry of the matrix of $\left.A^{n}\right|_{W}$ relative to the basis $\left\{\mathbf{v}_{1}, \ldots, \mathbf{v}_{t-1}\right\}$ is

$$
O\left(n^{t-2}|\lambda|^{n}\right)=O\left(n^{\ell-1} \rho^{n}\right)=o\left(n^{\ell} \rho^{n}\right),
$$

so we find that

$$
\left.\lim _{n \rightarrow \infty} n^{-\ell} \rho^{-n} A^{n}\right|_{W}=0 .
$$

On the other hand, the action of $A^{n}$ on the generator $\mathbf{v}_{t}$ of $V / W$ is given by

$A^{n} \mathbf{v}_{t}=\lambda^{n} \mathbf{v}_{t}+\left(\begin{array}{c}n \\ 1\end{array}\right) \lambda^{n-1} \mathbf{v}_{t-1}+\left(\begin{array}{l}n \\ 2\end{array}\right) \lambda^{n-2} \mathbf{v}_{t-2}+\cdots+\left(\begin{array}{c}n \\ t-1\end{array}\right) \lambda^{n-(t-1)} \mathbf{v}_{1}$.

The final term grows fastest at $n \rightarrow \infty$, so using the assumption that $t=\ell+1$, we find that

$$
n^{-\ell} \rho^{-n} A^{n} \mathbf{v}_{t}=\frac{1}{\lambda^{\ell} \ell !}\left(\frac{\lambda}{\rho}\right)^{n} \mathbf{v}_{1}+O\left(n^{-1}\right)
$$


We recall that $\mathcal{N} \subset \mathbb{N}$ is a sequence such that $n^{-\ell} \rho^{-n} A^{n}$ converges, so using the fact that $\lambda / \rho$ has magnitude 1 , we see that

$$
\lim _{n \in \mathcal{N}} n^{-\ell} \rho^{-n} A^{n} \mathbf{v}_{t}=\frac{\xi}{\rho^{\ell} \ell !} \mathbf{v}_{1} \quad \text { for some } \xi \in \mathbb{C} \text { with }|\xi|=1 .
$$

In particular, the limit is not 0 . Thus the action of $B$ of $V \otimes_{\overline{\mathbb{Q}}} \mathbb{C}$ satisfies

$$
\left.B\right|_{W \otimes_{\bar{Q}} \mathbb{C}}=0 \quad \text { and }\left.B\right|_{(V / W) \otimes_{\overline{\mathbb{Q}}} \mathbb{C}} \neq 0 .
$$

Hence

$$
\operatorname{ker}\left(\left.B\right|_{V \otimes_{\overline{\mathbb{Q}}} \mathbb{C}}\right)=W \otimes_{\overline{\mathbb{Q}}} \mathbb{C}
$$

where $W \otimes_{\overline{\mathbb{Q}}} \mathbb{C}$ is an $A$-invariant codimension 1 subspace of $V \otimes_{\overline{\mathbb{Q}}} \mathbb{C}$.

We now write $\overline{\mathbb{Q}}^{N}$ as an (internal) direct sum of $A$-invariant subspaces

$$
\overline{\mathbb{Q}}^{N}=V_{1} \dot{+} V_{2} \dot{+} \cdots \dot{+} V_{r} \dot{+} Z
$$

where $V_{1}, \ldots, V_{r}$ are the distinct maximal Jordan subspaces for $A$ and where $Z$ is the direct sum of all of the other Jordan subspaces for $A$. By definition, we have $r=r(A)$. Further, for each $i$ we let $W_{i} \subset V_{i}$ be the $A$-invariant codimension $1 \overline{\mathbb{Q}}$-subspace of $V_{i}$ satisfying

$$
\operatorname{ker}\left(\left.B\right|_{V_{i} \otimes_{\bar{Q}} \mathbb{C}}\right)=W_{i} \otimes_{\overline{\mathbb{Q}}} \mathbb{C}
$$

as described in (25). Since we also have $\operatorname{ker}\left(\left.B\right|_{Z \otimes_{\overline{\mathbb{Q}}} \mathbb{C}}\right)=0$ from (23), we see that the kernel of $B$ acting on $\mathbb{C}^{N} \cong \overline{\mathbb{Q}}^{N} \otimes_{\overline{\mathbb{Q}}} \mathbb{C}$ is

$$
\begin{aligned}
\operatorname{ker}_{\mathbb{C}}(B) & =\left(W_{1} \otimes_{\overline{\mathbb{Q}}} \mathbb{C}\right) \dot{+} \cdots \dot{+}\left(W_{r} \otimes_{\overline{\mathbb{Q}}} \mathbb{C}\right) \dot{+}\left(Z \otimes_{\overline{\mathbb{Q}}} \mathbb{C}\right) \\
& =\left(W_{1} \dot{+} \cdots+W_{r} \dot{+} Z\right) \otimes_{\overline{\mathbb{Q}}} \mathbb{C} .
\end{aligned}
$$

To ease notation, we let

$$
U=W_{1} \dot{+} \cdots \dot{+} W_{r} \dot{+} Z \subset \overline{\mathbb{Q}}^{N},
$$

so $U$ is a $\overline{\mathbb{Q}}$-vector space satisfying

$$
\operatorname{ker}_{\mathbb{C}}(B)=U \otimes_{\overline{\mathbb{Q}}} \mathbb{C} \quad \text { and } \quad \operatorname{dim}_{\overline{\mathbb{Q}}} U=N-r .
$$

The dimension of $U$ follows from the fact that each $W_{i}$ has codimension 1 in $V_{i}$, and $V_{1} \dot{+} \cdots \dot{+} V_{r} \dot{+} Z=\overline{\mathbb{Q}}^{N}$. We also note that $U$ depends only on the matrix $A$, which is clear from above, or from the alternative description of $U$ as the kernel of the linear transformation ${ }^{6}$

$$
\prod_{|\lambda|<\rho}(A-\lambda)^{N} \cdot \prod_{|\lambda|=\rho}(A-\lambda)^{\ell} \in \operatorname{Mat}_{N}(\overline{\mathbb{Q}}) .
$$

Returning to our point $P \in \mathbb{G}_{m}^{N}(\overline{\mathbb{Q}})$ with height $\hat{h}_{\varphi_{A}}(P)=0$, we next observe that

$$
\operatorname{Perp}_{\mathbb{C}}\left(\log \|P\|_{v}\right) \supset \operatorname{Perp}_{\mathbb{C}}\left(\operatorname{ker}_{\mathbb{C}}(B)\right) \quad \text { from }(22),
$$

\footnotetext{
${ }^{6}$ For further details, see Appendix A.5.
} 


$$
\begin{array}{ll}
=\operatorname{Perp}_{\mathbb{C}}\left(U \otimes_{\overline{\mathbb{Q}}} \mathbb{C}\right) & \text { from }(27), \\
=\operatorname{Perp}_{\overline{\mathbb{Q}}}(U) \otimes_{\overline{\mathbb{Q}}} \mathbb{C} & \text { from Lemma 34(a), } \\
\supset \operatorname{Perp}_{\overline{\mathbb{Q}}}(U) . &
\end{array}
$$

This gives us some vectors in $\overline{\mathbb{Q}}^{N}$ that annihlate log $\|P\|_{v}$, but our goal is to find vectors in $\mathbb{Q}^{N}$ with this property. We note that the coordinates of the vector $\log \|P\|_{v}$ are logarithms of algebraic numbers, so we can apply Baker's theorem (Theorem 35) to conclude that the $\overline{\mathbb{Q}}$ vector space $\operatorname{Perp}_{\overline{\mathbb{Q}}}\left(\log \|P\|_{v}\right)$ is Galois invariant as a subspace of $\overline{\mathbb{Q}}^{N}$. Hence (28) implies that

$$
\operatorname{Perp}_{\overline{\mathbb{Q}}}\left(\log \|P\|_{v}\right) \supset \sum_{\sigma \in \operatorname{Gal}(\overline{\mathbb{Q}} / \mathbb{Q})} \sigma\left(\operatorname{Perp}_{\overline{\mathbb{Q}}}(U)\right) .
$$

The $\overline{\mathbb{Q}}$-vector space on the right-hand side of $(29)$ is $\operatorname{Gal}(\overline{\mathbb{Q}} / \mathbb{Q})$-invariant, so Lemma 34 (b) says that there is a (unique) $\mathbb{Q}$-vector space $Y \subset$ $\mathbb{Q}^{N}$ such that

$$
\sum_{\sigma \in \operatorname{Gal}(\overline{\mathbb{Q}} / \mathbb{Q})} \sigma\left(\operatorname{Perp}_{\overline{\mathbb{Q}}}(U)\right)=Y \otimes_{\mathbb{Q}} \overline{\mathbb{Q}} .
$$

We stress here that $Y$ depends only on $A$ and is independent of $P$ and $v$, since the same is true of $U$. It follows from (29) that

$$
\operatorname{Perp}_{\mathbb{Q}}\left(\log \|P\|_{v}\right) \supset Y .
$$

We defer the computation of $\operatorname{dim}_{\mathbb{Q}}(Y)$, which turns out to equal $\bar{r}$, until the end of the proof.

We let

$$
L=Y \cap \mathbb{Z}^{N} \subset \mathbb{Z}^{N},
$$

so $L$ is an integral lattice satisfying

$$
\operatorname{rank}_{\mathbb{Z}} L=\operatorname{dim}_{\mathbb{Q}} Y .
$$

It follows from (31) that

$$
\mathbf{e} \cdot \log \|P\|_{v}=0 \quad \text { for all } \mathbf{e} \in L .
$$

Writing

$$
P=\left(x_{1}, \ldots, x_{N}\right) \in \mathbb{G}_{m}^{N}(K) \text { and } \mathbf{e}=\left(e_{1}, \ldots, e_{N}\right) \in L,
$$

\footnotetext{
${ }^{7}$ If $v$ is non-archimedean, then $\left\|x_{i}\right\|_{v}=p_{v}^{r_{i, v}}$ for a rational prime $p_{v}$ and rational numbers $r_{i, v} \in \mathbb{Q}$, so $\log \|P\|_{v}$ is a vector in $\mathbb{Q}^{N}$ multiplied by the scalar $\log p_{v}$. Thus in the non-archimedean case, the equality $\operatorname{Perp}_{\overline{\mathbb{Q}}}\left(\log \|P\|_{v}\right)=\operatorname{Perp}_{\mathbb{Q}}\left(\log \|P\|_{v}\right) \otimes_{\mathbb{Q}} \overline{\mathbb{Q}}$ is a triviality. But for archimedean $v$, we appear to need the full strength of Baker's theorem.
} 
this becomes

$$
\prod_{j=1}^{N}\left\|x_{j}\right\|_{v}^{e_{j}}=\left\|\prod_{j=1}^{N} x_{j}^{e_{j}}\right\|_{v}=1
$$

where note that we are allowed to move the $e_{j}$ across the absolute value signs because they are integers. Formula (32) holds for all $v \in M_{K}$, so Kronecker's theorem [57, Theorem 3.8] implies that

$$
\prod_{j=1}^{N} x_{j}^{e_{j}} \quad \text { is a root of unity for all } \mathbf{e} \in L .
$$

We stress that $(33)$ holds for all points $P \in \mathbb{G}_{m}^{N}(\overline{\mathbb{Q}})$ satsifying $\hat{h}_{\varphi_{A}}(P)=$ 0 and for all $\mathbf{e} \in L$, where $L$ is independent of $P$.

The lattice $L$ is associated to an algebraic subgroup $G_{L}$ of $\mathbb{G}_{m}^{N}$ in the usual way,

$$
G_{L}=\bigcap_{\mathbf{e} \in L}\left\{X_{1}^{e_{1}} \cdots X_{N}^{e_{N}}=1\right\}
$$

and the dimension of $G_{L}$ is given by

$$
\operatorname{dim} G_{L}=N-\operatorname{rank}_{\mathbb{Z}} L=N-\operatorname{dim}_{\mathbb{Q}} Y .
$$

Further, we see from (33) that if $\hat{h}_{\varphi_{A}}(P)=0$, then some power of the coordinates of $P$ gives a point in $G_{L}(\overline{\mathbb{Q}})$, which shows that

$$
\left\{P \in \mathbb{G}_{m}^{N}(\overline{\mathbb{Q}}): \hat{h}_{\varphi_{A}}(P)=0\right\} \subset G_{L}(\overline{\mathbb{Q}})^{\text {div }} .
$$

It remains to prove that $\operatorname{dim} G_{L}=N-\bar{r}$, which from (35), is equivalent to showing that $\operatorname{dim}_{\mathbb{Q}} Y=\bar{r}$. We compute

$$
\begin{aligned}
\operatorname{dim}_{\mathbb{Q}} Y & =\operatorname{dim}_{\overline{\mathbb{Q}}}\left(\sum_{\sigma \in \operatorname{Gal}(\overline{\mathbb{Q}} / \mathbb{Q})} \sigma\left(\operatorname{Perp}_{\overline{\mathbb{Q}}}(U)\right)\right) \quad \text { from }(30), \\
& =\operatorname{dim}_{\overline{\mathbb{Q}}}\left(\sum_{\sigma \in \operatorname{Gal}(\overline{\mathbb{Q}} / \mathbb{Q})} \operatorname{Perp}_{\overline{\mathbb{Q}}}(\sigma U)\right) \\
& =\operatorname{dim}_{\overline{\mathbb{Q}}} \operatorname{Perp}_{\overline{\mathbb{Q}}}\left(\bigcap_{\sigma \in \operatorname{Gal}(\overline{\mathbb{Q}} / \mathbb{Q})} \sigma U\right) \quad \text { from Lemma 34(d), } \\
& =N-\operatorname{dim}_{\overline{\mathbb{Q}}}\left(\bigcap_{\sigma \in \operatorname{Gal}(\overline{\mathbb{Q}} / \mathbb{Q})} \sigma U\right) \\
& =N-\operatorname{dim}_{\overline{\mathbb{Q}}}\left(\bigcap_{\sigma \in \operatorname{Gal}(\overline{\mathbb{Q}} / \mathbb{Q})}\left(\sum_{i=1}^{r} \sigma W_{i} \dot{+} \sigma Z\right)\right) \quad \text { from }(26) .
\end{aligned}
$$


Each $W_{i}$ is a codimension one subspace of a maximal Jordan block $V_{i}$, so each distinct Galois conjugate of a maximal Jordan block contributes codimension one to the intersection. By definition, the number of such conjugates is $\bar{r}$, so the codimension of the intersection is $\bar{r}$, and hence the dimension of the intersection is is $N-\bar{r}$. This proves that $\operatorname{dim}_{\mathbb{Q}} Y=$ $\bar{r}$, which completes the proof of Theorem 27 .

\section{Proof of Corollaries of Theorem 27}

In this section we give the proofs of Corollaries 29, 31, 32, and 33 to Theorem 27.

Proof of Corollary 29. For $Q=\left(y_{1}, \ldots, y_{N}\right) \in \mathbb{G}_{m}^{N}(\overline{\mathbb{Q}})$ and $d \in \mathbb{Z}$, we use the notation

$$
Q^{d}=\left(y_{1}^{d}, \ldots, y_{N}^{d}\right)
$$

We let $G \subsetneq \mathbb{G}_{m}^{N}$ be the algebraic subgroup described in the statement of Theorem 27 for the monomial map $\varphi$, and we let $L \subset \mathbb{Z}^{N}$ be the lattice associated to $G$ via (34).

The assumption that $\hat{h}_{\varphi}(P)=0$ implies that

$$
\hat{h}_{\varphi}\left(\varphi^{n}(P)\right)=\delta_{\varphi}^{n} \hat{h}_{\varphi}(P)=0 \text { for all } n \geq 0,
$$

so Theorem 27 says that $\varphi^{n}(P) \subset G(\overline{\mathbb{Q}})^{\text {div }}$ for all $n \geq 0$. However, the points $\varphi^{n}(P)$ in the orbit $\mathcal{O}_{\varphi}(P)$ are all defined over the number field $K=\mathbb{Q}(P)$, so

$$
\mathcal{O}_{\varphi}(P) \subset G(\overline{\mathbb{Q}})^{\operatorname{div}} \cap \mathbb{G}_{m}^{N}(K) .
$$

Let $d$ be the number of roots of unity in $K$. We are going to prove that

$$
\mathcal{O}_{\varphi}(P)^{d} \subset G(K) \text {. }
$$

This will complete the proof, since writing $G=G_{L}$ to indicate the dependence of $G$ on the lattice $L \subset \mathbb{Z}^{N}$, we clearly have

$$
\left\{Q \in \mathbb{G}_{m}^{N}(\overline{\mathbb{Q}}): Q^{d} \in G_{L}(\overline{\mathbb{Q}})\right\}=G_{d L}(\overline{\mathbb{Q}}) .
$$

So (38) implies that $\mathcal{O}_{\varphi}(P) \subset G_{d L}(K)$. But $G_{d L}$ is an algebraic subgroup of $\mathbb{G}_{m}^{N}$ of the same dimension as $G_{L}$, and hence $\mathcal{O}_{\varphi}(P)$ is contained in a proper algebraic subgroup of $\mathbb{G}_{m}^{N}$.

To prove the claim, let

$$
Q=\left(y_{1}, \ldots, y_{N}\right) \in G(\overline{\mathbb{Q}})^{\operatorname{div}} \cap \mathbb{G}_{m}^{N}(K) .
$$

The assumption that $Q \in G(\overline{\mathbb{Q}})^{\text {div }}$ means that there is an $m \geq 1$ such that $Q^{m} \in G(\overline{\mathbb{Q}})$; we take the smallest such $m$. The group $G$ is defined by the lattice $L$, so

$$
y_{1}^{e_{1} m} \cdots y_{N}^{e_{N} m}=1 \quad \text { for all } \mathbf{e}=\left(e_{1}, \ldots, e_{N}\right) \in L .
$$


Taking roots, this implies that

$$
y_{1}^{e_{1}} \cdots y_{N}^{e_{N}} \text { is an } m^{\text {th }} \text {-root of unity for all } \mathbf{e} \in L \text {. }
$$

But $Q$ is in $\mathbb{G}_{m}^{N}(K)$, so $y_{1}^{e_{1}} \cdots y_{N}^{e_{N}} \in K$, and hence $y_{1}^{e_{1}} \cdots y_{N}^{e_{N}}$ is a $d^{\text {th }}$ root of unity. It follows that $m \mid d$, so in particular $Q^{d} \in G(\overline{\mathbb{Q}})$. But also $Q \in \mathbb{G}_{m}^{N}(K)$, so $Q^{d} \in G(K)$. This is true for every $Q \in$ $G(\overline{\mathbb{Q}})^{\operatorname{div}} \cap \mathbb{G}_{m}^{N}(K)$, so $(37)$ implies that $Q^{d} \in G(K)$ for every $Q \in \mathcal{O}_{\varphi}(P)$. Hence $\mathcal{O}_{\varphi}(P)^{d} \in G(K)$, which completes the proof of (38), and with it the proof of Corollary 29.

Proof of Corollary 31. We already know that

$$
P \in \operatorname{PrePer}(\varphi) \quad \Longrightarrow \quad \hat{h}_{\varphi}(P)=0
$$

from Proposition 19(c), so we assume that $\hat{h}_{\varphi}(P)=0$, and we want to prove that $P$ is preperiodic for $\varphi$.

We have assumed that $\operatorname{det}(T-A)$ is irreducible, so the eigenvalues $\lambda_{1}, \ldots, \lambda_{N}$ of $A$ are distinct and form a complete set of Galois conjugates. Hence the Jordan subspaces are all 1-dimensional and are pairwise Galois conjugate. Since at least one of them is a maximal Jordan subspace, we see that $\bar{r}(A)=N$, and hence the algebraic subgroup $G \subset \mathbb{G}_{m}^{N}$ described in Theorem 27 has dimension 0. It follows that the divisible hull of $G$ is given by

$$
G(\overline{\mathbb{Q}})^{\operatorname{div}}=\mathbb{G}_{m}^{N}(\overline{\mathbb{Q}})_{\text {tors }},
$$

where the torsion subgroup $\mathbb{G}_{m}^{N}(\overline{\mathbb{Q}})_{\text {tors }}$ consists of all points whose coordinates are roots of unity. Theorem 27 and the assumption that $\hat{h}_{\varphi}(P)=0$ imply that $P \in G(\overline{\mathbb{Q}})^{\text {div }}$. It is then clear that $P$ is preperiodic for the monomial map $\varphi$, since the coordinates of $\varphi^{n}(P)$ are all roots of unity lying in the number field $\mathbb{Q}(P)$, so take on only finitely many possible values.

Proof of Corollary 32. If $\delta_{\varphi}=1$, then Proposition 12 tells us that $1 \leq$ $\alpha_{\varphi}(P) \leq \delta_{\varphi}=1$, so $\alpha_{\varphi}(P)=1$ for all points $P$. We assume for the remainder of the proof that $\delta_{\varphi}>1$.

The following fact will be useful later in the proof. Letting $P^{d}=$ $\left(x_{1}^{d}, \ldots, x_{N}^{d}\right)$ and noting that $\varphi$ is a homomorphism of $\mathbb{G}_{m}^{N}$, we have

$$
h\left(\varphi^{n}\left(P^{d}\right)\right)=h\left(\varphi^{n}(P)^{d}\right)=d h\left(\varphi^{n}(P)\right) .
$$

It then follows directly from the definitions of canonical height and arithmetic degree that

$$
\hat{h}_{\varphi}\left(P^{d}\right)=d \hat{h}_{\varphi}(P) \quad \text { and } \quad \alpha_{\varphi}\left(P^{d}\right)=\alpha_{\varphi}(P) \quad \text { for any } d \geq 1 .
$$

Let $G \subset \mathbb{G}_{m}^{N}$ be the smallest algebraic subgroup of $\mathbb{G}_{m}^{N}$ that contains the orbit $\mathcal{O}_{\varphi}(P)$. The group $G$ might not be connected, but its identity 
component $G_{0}$ has finite index in $G$, say $d=\left(G: G_{0}\right)$. Then the orbit of $P^{d}$ is contained in $G_{0}$, so the smallest algebraic subgroup of $\mathbb{G}_{m}^{N}$ containing $\mathcal{O}_{\varphi}\left(P^{d}\right)$ is $G_{0}$. Since $\alpha_{\varphi}\left(P^{d}\right)=\alpha_{\varphi}(P)$ from (39), we may replace $P$ with $P^{d}$, which reduces us to the case that the group $G$ is connected.

We next note that since $\varphi$ is a homomorphism of $\mathbb{G}_{m}^{N}$, the smallest algebraic subgroup $G$ containing the orbit $\mathcal{O}_{\varphi}(P)$ is itself $\varphi$ invariant, i.e., $\varphi(G) \subset G$. ${ }^{8}$ The group $G$ corresponds to a lattice $L \subset \mathbb{Z}^{N}$. More precisely, there is a perfect pairing

$$
\mathbb{Z}^{N} \times \mathbb{G}_{m}^{N} \longrightarrow \mathbb{G}_{m}, \quad(\mathbf{e}, \mathbf{x}) \longmapsto \mathbf{x}^{\mathbf{e}}=\prod_{i=1}^{N} x_{i}^{e_{i}},
$$

and $G_{L}$ is by definition the right kernel of $L$ for this pairing. The inclusion $\varphi\left(G_{L}\right) \subset G_{L}$ is equivalent to the inclusion $L A \subset A$, where $A$ is the matrix associated to the monomial map $\varphi$, and the restriction of $\varphi$ to $G_{L}$ is a monomial map whose associated linear transformation (over $\mathbb{Q}$ ) is the restriction of $A$ to $L^{\perp}$, where

$$
L^{\perp}=\left\{\mathbf{f} \in \mathbb{Z}^{N}: \mathbf{f} \cdot \mathbf{e}=0 \text { for all } \mathbf{e} \in L\right\} .
$$

More precisely, there is a finite homomorphism $\mathbb{G}_{m}^{N-k} \rightarrow G_{L}$ such that $\varphi$ induces a monomial map $\mathbb{G}_{m}^{N-k} \rightarrow \mathbb{G}_{m}^{N-k}$ whose associated linear transformation (extended to $\mathbb{Q}$ ) is isomorphic to the restriction of $A$ to $\Lambda^{\perp} \otimes \mathbb{Q}$. We write $\varphi_{L}$ for the induced monomial map on $\mathbb{G}_{m}^{N-k}$, we let $A_{L}^{\perp}$ be the linear transformation that $A$ induces on $L^{\perp} \otimes \mathbb{Q}$, and we replace $P$ by a point in $\mathbb{G}_{m}^{N-k}$ that maps to $P$.

We apply Corollary 29 to the $\left.\varphi\right|_{L^{-}}$orbit of $P$. We chose $G_{L}$ to be the smallest algebraic subgroup of $\mathbb{G}_{m}^{N}$ that contains $\mathcal{O}_{\varphi}(P)$, so Corollary 29 tells us that $\hat{h}_{\varphi_{L}, G_{L}}(P)>0$. On the other hand, we have the implications

$$
\begin{aligned}
\hat{h}_{\varphi_{L}, G_{L}}(P)>0 & \Longrightarrow \alpha_{\varphi_{L}, G_{L}}(P)=\delta_{\varphi_{L}, G_{L}} \quad \text { from Proposition 19(d), } \\
& \Longrightarrow \alpha_{\varphi_{L}, G_{L}}(P)=\rho\left(A_{L}^{\perp}\right) \quad \text { from Proposition 21(c). }
\end{aligned}
$$

The characteristic polynomial of $A_{L}^{\perp}$ as a linear transormation of $L^{\perp} \otimes \mathbb{Q}$ is a polynomial in $\mathbb{Q}[T]$ that divides the characteristic polynomial of $A$ as a linear transformation of $\mathbb{Q}^{N}$, and hence $\rho\left(A_{L}^{\perp}\right)$ is the largest root of some factor of $\operatorname{det}(T-A)$ in $\mathbb{Q}[T]$. We also note that $\alpha_{\varphi_{L}, G_{L}}(P)=$ $\alpha_{\varphi}(P)$, since for any morphism $i: \mathbb{P}^{k} \rightarrow \mathbb{P}^{N}$ with $\operatorname{dim} i\left(\mathbb{P}^{k}\right)=k$,

\footnotetext{
${ }^{8}$ For further details, see Appendix A.6.
} 
we have $h_{\mathbb{P}^{N}} \circ i \asymp h_{\mathbb{P}^{k}}$, so we can compute the arithmetic degree on either $\mathbb{G}_{m}^{N}$ or $G_{L} \cdot{ }^{9}$ This proves that

$$
\left\{\alpha_{\varphi}(P): P \in \mathbb{G}_{m}^{N}(\overline{\mathbb{Q}})\right\} \subset\left\{1, \rho\left(f_{1}\right), \rho\left(f_{2}\right), \ldots, \rho\left(f_{s}\right)\right\} .
$$

For the opposite inclusion, let $f(T) \in \mathbb{Z}[T]$ be a monic irreducible factor of the characteristic polynomial of $A$ with $\rho(f)>1$, and write

$$
\operatorname{det}(T-A)=f(T)^{e} g(T) \quad \text { with } f(T) \nmid g(T) .
$$

Evaluation at $\varphi$ gives a map $\mathbb{Z}[T] \rightarrow \operatorname{End}\left(\mathbb{G}_{m}^{N}\right)$, i.e.,

$$
\left(\sum b_{i} T^{i}\right) \cdot P=\prod \varphi^{i}(P)^{b_{i}}
$$

In particular, note that $\operatorname{det}(T-A)$ annihliates $\mathbb{G}_{m}^{N}$, since a linear transformation is a root of its own characteristic polynomial. Consider the subgroup of $\mathbb{G}_{m}^{N}$ defined by

$$
G=g(T) \cdot \mathbb{G}_{m}^{N}
$$

Then $G$ is a nontrivial $\varphi$-invariant algebraic subgroup of $\mathbb{G}_{m}^{N}$ satisfying $f(T)^{e} \cdot G=0$. The group $G$ is a torus, and the monomial map $\varphi_{G}: G \rightarrow G$ satisfies $f\left(\varphi_{G}\right)^{e}=1$, so the associated matrix $A_{G}$ satisfies $f\left(A_{G}\right)^{e}=1$. Since $f$ is irreducible, it follows that the characteristic polynomial of $A_{G}$ is a positive power of $f(T)$, and then Proposition 21(c) tells us that $\delta_{\varphi_{G}}=\rho\left(A_{G}\right)=\rho(f)$. Further, if we take any $P \in G(\overline{\mathbb{Q}})$ whose orbit $\mathcal{O}_{\varphi}(P)$ is Zariski dense in $G$, then Corollary 29 tells us that $\hat{h}_{G,\left.\varphi\right|_{G}}(P)>0$, and then Proposition $19(\mathrm{~d})$ says that

$$
\alpha_{\varphi}(P)=\alpha_{G,\left.\varphi\right|_{G}}(P)=\delta_{\varphi_{G}}=\rho(f) .
$$

Since we also always have $\alpha_{\varphi}(1)=1$, this completes the proof of the other inclusion

$$
\left\{\alpha_{\varphi}(P): P \in \mathbb{G}_{m}^{N}(\overline{\mathbb{Q}})\right\} \supset\left\{1, \rho\left(f_{1}\right), \rho\left(f_{2}\right), \ldots, \rho\left(f_{s}\right)\right\},
$$

and with it, the proof of Corollary 32.

Proof of Corollary 33. The implication $\hat{h}_{\varphi}(P)>0 \Rightarrow \alpha_{\varphi}(P)=\delta_{\varphi}$ is easy to prove for all dominant rational maps; see Proposition 19(d). So we need to prove the opposite implication under the assumption that $A$ is diagonalizable. ${ }^{10}$ We note that the diagonalizability condition implies in particular that $\ell(A)=0$, since every Jordan block has dimension 1 .

Let $P$ be a point with $\hat{h}_{\varphi}(P)=0$. We continue with the notation from the proof of Corollary 32, so in particular $G=G_{L} \subset \mathbb{G}_{m}^{N}$ is the

\footnotetext{
${ }^{9}$ For further details, see Appendix A.7.

${ }^{10}$ For further details, see Appendix A.8.
} 
smallest algebraic subgroup containing $\mathcal{O}_{\varphi}(P)$ and $\varphi_{L}$ is the restriction of $\varphi$ to $G_{L}$. We proved that

$$
\alpha_{\varphi}(P)=\alpha_{\varphi_{L}, G_{L}}(P)=\rho\left(A_{L}^{\perp}\right)=\delta_{\varphi_{L}} ;
$$

see (40) and the remark following for the first two equalities, while the third equality is Proposition 21(c). The assumption that $G_{L}$ is the smallest algebraic subgroup containing $\mathcal{O}_{\varphi}(P)$, combined with Corollary 29, implies that $\hat{h}_{G_{L}, \varphi_{L}}(P)>0$. Using the definition of canonical height, we have

$$
\begin{aligned}
& 0=\hat{h}_{\varphi}(P)=\lim _{n \rightarrow \infty} \frac{1}{\delta_{\varphi}^{n}} h\left(\varphi^{n}(P)\right), \\
& 0<\hat{h}_{\varphi_{L}, G_{L}}(P)=\lim _{n \rightarrow \infty} \frac{1}{\delta_{\varphi_{L}}^{n}} h\left(\varphi^{n}(P)\right) .
\end{aligned}
$$

It follows that $\delta_{\varphi_{L}}<\delta_{\varphi}$ (strict inequality), which together with (41) gives the desired inequality $\alpha_{\varphi}(P)<\delta_{\varphi}$.

\section{Regular afFine automorphisms}

A rational map is algebraically stable if $\operatorname{deg}\left(\varphi^{n}\right)$ is equal to $(\operatorname{deg} \varphi)^{n}$ for all $n \geq 1$, which from Proposition 7 is equivalent to $\delta_{\varphi}=\operatorname{deg} \varphi$. As noted in Section 2.2, regular affine automorphisms are algebraically stable. In this section we describe Kawaguchi's theory of canonical heights and use it to illustrate some of the ideas in this paper and to raise a question about birational maps.

Theorem 36. Let $\varphi: \mathbb{A}^{N} \rightarrow \mathbb{A}^{N}$ be a regular affine automorphism, i.e., the extensions of $\varphi$ and $\varphi^{-1}$ to $\mathbb{P}^{N}$ are not morphisms, but satisfy $Z(\varphi) \cap Z\left(\varphi^{-1}\right)=\emptyset$. Let $\hat{h}_{\varphi}$ and $\hat{h}_{\varphi^{-1}}$ be the canonical heights associated to $\varphi$ and $\varphi^{-1}$, respectively.

(a) $\delta_{\varphi}=\operatorname{deg}(\varphi)$ and $\delta_{\varphi^{-1}}=\operatorname{deg}\left(\varphi^{-1}\right)$.

(b) $h(P)+O(1) \leq \hat{h}_{\varphi}(P)+\hat{h}_{\varphi^{-1}}(P) \leq 2 h(P)+O(1)$ for all $P \in \mathbb{A}^{N}(\overline{\mathbb{Q}})$.

(c) $\hat{h}_{\varphi}(P)=0 \Longleftrightarrow \hat{h}_{\varphi^{-1}}(P)=0 \Longleftrightarrow P \in \operatorname{Per}(\varphi)$.

Proof. (a) The fact that regular affine automorphisms are algebraically stable is well-known; see for example [54, Chapter 2].

(b,c) The construction is due to Kawaguchi [38] (see also [57, Exercises 7.17-7.22]), but at the time it was only known to work for $N=2$. The deep height inequality needed to justify the construction for general $N$ was proven independently by Kawaguchi [39] and Lee [42].

Using Theorem 36, it is easy to compute $\alpha_{\varphi}(P)$ for regular affine automorphisms. 
Corollary 37. Let $\varphi: \mathbb{P}^{N} \rightarrow \mathbb{P}^{N}$ be a regular affine automorphism defined over $\overline{\mathbb{Q}}$, and let $P \in \mathbb{A}^{N}(\overline{\mathbb{Q}})$. Then

$$
\alpha_{\varphi}(P)= \begin{cases}\delta_{\varphi} & \text { if } P \notin \operatorname{Per}(\varphi), \\ 1 & \text { if } P \in \operatorname{Per}(\varphi) .\end{cases}
$$

Proof. If $P \in \operatorname{Per}(\varphi)$, it is clear that $\alpha_{\varphi}(P)=1$. Conversely, if $P \notin$ $\operatorname{Per}(\varphi)$, then Theorem 36(c) says that $\hat{h}_{\varphi}(P)>0$, so Proposition 19 implies that $\alpha_{\varphi}(P)=\delta_{\varphi}$.

Remark 38. If $\varphi: \mathbb{P}^{N} \rightarrow \mathbb{P}^{N}$ is a dominant birational map with $\delta_{\varphi}>1$ and $\delta_{\varphi^{-1}}>1$, then there are canonical heights associated to both $\varphi$ and $\varphi^{-1}$. Neither of these canonical heights can individually satsify $\hat{h} \asymp h$, but Kawaguchi's construction suggests looking at the sum $\hat{h}_{\varphi}+\hat{h}_{\varphi^{-1}}$. It would be very interesting to give general conditons which imply that $\hat{h}_{\varphi}+\hat{h}_{\varphi^{-1}} \asymp h$, since it is an exercise to prove that ${ }^{11}$

$$
\hat{h}_{\varphi}+\hat{h}_{\varphi^{-1}} \asymp h \text { and } \quad \hat{h}_{\varphi}(P)=0 \quad \Longrightarrow \quad P \in \operatorname{Per}(\varphi) .
$$

\section{Dominant Self-Maps of General Varieties}

Up to now we have restricted attention to rational self-maps of $\mathbb{P}^{N}$. In this section we describe how Conjecture 1 may be extended to arbitrary varieties. As usual, for any endomorphism $F: V \rightarrow V$ of a finite dimensional $\mathbb{C}$-vector space, we write

$$
\rho(F)=\max \{|\lambda|: \lambda \text { is an eigenvalue of } F\}
$$

for the spectral radius of $F$.

Definition. Let $X$ be a nonsingular irreducible algebraic variety, and let $\psi: X \rightarrow X$ be a dominant rational map. Then $\psi$ induces a $\mathbb{Q}$ linear endomorphism $\psi^{*}$ of the rational Néron-Severi group $\mathrm{NS}(X)_{\mathbb{Q}}=$ $\mathrm{NS}(X) \otimes \mathbb{Q}$. N.B. In general, $\left(\psi_{1} \circ \psi_{2}\right)^{*} \neq \psi_{2}^{*} \circ \psi_{1}^{*}$. Let $\varphi: X \rightarrow X$ be a dominant rational map. The (first) dynamical degree of $\varphi$ is

$$
\delta_{\varphi}=\lim _{n \rightarrow \infty} \rho\left(\left(\varphi^{n}\right)^{*}\right)^{1 / n} .
$$

We note that if $X=\mathbb{P}^{N}$, then $\operatorname{NS}(X)_{\mathbb{Q}}=\mathbb{Q}$ and $\rho\left(\varphi^{*}\right)=\operatorname{deg}(\varphi)$, so this definition is consistant with our earlier definition.

Definition. Let $X$ be a nonsingular irreducible algebraic variety defined over $\overline{\mathbb{Q}}$, let $\varphi: X \rightarrow X$ be a dominant rational map defined

\footnotetext{
${ }^{11}$ For further details, see Appendix A.9.
} 
over $\overline{\mathbb{Q}}$, and fix a height function $h_{X}$ on $X(\overline{\mathbb{Q}})$ associated to an ample divisor. Also let

$$
X(\overline{\mathbb{Q}})_{\varphi}=\left\{P \in X(\overline{\mathbb{Q}}): \mathcal{O}_{\varphi}(P) \cap Z(\varphi)=\emptyset\right\} .
$$

Then for $P \in X(\overline{\mathbb{Q}})_{\varphi}$, we define the arithmetic degree of $\varphi$ at $P$ to be

$$
\alpha_{\varphi}(P)=\limsup _{n \rightarrow \infty} h_{X}\left(\varphi^{n}(P)\right)^{1 / n} .
$$

Remark 39. The definition of $\alpha_{\varphi}(P)$ is independent of the choice of height function on $X$, because if $D$ and $E$ are ample divisors on $X$, then $h_{X, D} \asymp h_{X, E}$. Hence there is a constant $C>0$ such that

$$
C^{-1} h_{X, E}\left(\varphi^{n}(P)\right)-C \leq h_{X, D}\left(\varphi^{n}(P)\right) \leq C h_{X, E}\left(\varphi^{n}(P)\right)+C .
$$

Taking the $n^{\text {th }}$-root and letting $n \rightarrow \infty$ shows that $h_{X, D}$ and $h_{X, E}$ yield the same value of $\alpha_{\varphi}(P)$.

We now generalize Conjecture 1.

Conjecture 40. Let $X$ be a nonsingular irreducible algebraic variety defined over $\overline{\mathbb{Q}}$, and let $\varphi: X \rightarrow X$ be a dominant rational map defined over $\overline{\mathbb{Q}}$.

(a) The set

$$
\left\{\alpha_{\varphi}(P): P \in X(\overline{\mathbb{Q}})_{\varphi}\right\}
$$

is a finite set of algebraic integers.

(b) Let $P \in X(\overline{\mathbb{Q}})_{\varphi}$ be a point such that $\mathcal{O}_{\varphi}(P)$ is Zariski dense in $X$. Then $\alpha_{\varphi}(P)=\delta_{\varphi}$.

If $\varphi$ is a morphism, then $\rho\left(\varphi^{* n}\right)=\rho\left(\varphi^{*}\right)^{n}$, so in particular $\delta_{\varphi}=\rho\left(\varphi^{*}\right)$ is an algebraic integer. However, even for morphisms, Conjecture 40 appears to be nontrivial in general. We now show that it is true for the K3 surfaces and automorphisms studied in [55].

Theorem 41. Let $X \subset \mathbb{P}^{2} \times \mathbb{P}^{2}$ be a smooth surface given by the intersection of $a(2,2)$-form and $a(1,1)$-form, and assume that $\mathrm{NS}(X) \cong$ $\mathbb{Z}^{2}$. The two projections $\pi_{1}, \pi_{2}: X \rightarrow \mathbb{P}^{2}$ induce noncommuting involutions $\iota_{1}, \iota_{2}: X \rightarrow \mathbb{P}^{2}$, and the map $\varphi=\iota_{1} \circ \iota_{2}$ is a automorphism of $X$ of infinite order. (See [55] for details.) Then $\delta_{\varphi}=7+4 \sqrt{3}$,

$$
\alpha_{\varphi}(P)=\delta_{\varphi} \Longleftrightarrow P \notin \operatorname{Per}(\varphi) \Longleftrightarrow \mathcal{O}_{\varphi}(P) \text { is Zariski dense },
$$

and

$$
\alpha_{\varphi}(P)=1 \Longleftrightarrow P \in \operatorname{Per}(\varphi) \Longleftrightarrow \mathcal{O}_{\varphi}(P) \text { is not Zariski dense. }
$$

In particular, Conjecture 40 is true. 
Proof. To ease notation, let $\beta=7+4 \sqrt{3}$. It is shown in $[55]$ that $\varphi^{*}$ acts on the natural basis $\left\{\pi_{1}^{*} H, \pi_{2}^{*} H\right\}$ of $\operatorname{Pic}(X)=\mathrm{NS}(X)$ via the matrix $\left(\begin{array}{ll}-1 & 4 \\ -4 & 15\end{array}\right)$. This matrix has eigenvalues $\beta$ and $\beta^{-1}$, and $\varphi$ is a morphism, so $\delta_{\varphi}=\beta$.

It is further shown that there are divisors $E^{+}, E^{-} \in \mathrm{NS}(X) \otimes \mathbb{R}$ satisfying $\varphi^{*} E^{+}=\beta E^{+}$and $\varphi^{*} E^{-}=\beta^{-1} E^{-}$and such that $E^{+}+E^{-}$ is in the ample cone. Writing the associated canonical height functions as $\hat{h}^{+}$and $\hat{h}^{-}$, the function $\hat{h}=\hat{h}^{+}+\hat{h}^{-}$is a Weil height function associated to an ample divisor, so we can use it to compute $\alpha_{\varphi}(P)$. In particular, it is proven in [55] that

$$
\begin{aligned}
\hat{h}^{+}(P)=0 \Longleftrightarrow \hat{h}^{-}(P)=0 & \Longleftrightarrow \hat{h}(P)=0 \Longleftrightarrow P \in \operatorname{Per}(\varphi) \\
& \Longleftrightarrow \mathcal{O}_{\varphi}(P) \text { is not Zariski dense. }
\end{aligned}
$$

It is clear that if $P \in \operatorname{Per}(\varphi)$, then $\alpha_{\varphi}(P)=1$. Suppose now that $P \notin$ $\operatorname{Per}(\varphi)$, or equivalently, that $\mathcal{O}_{\varphi}(P)$ is Zariski dense. Then

$$
\hat{h}\left(\varphi^{n}(P)\right)=\hat{h}^{+}\left(\varphi^{n}(P)\right)+\hat{h}^{-}\left(\varphi^{n}(P)\right)=\beta^{n} \hat{h}^{+}(P)+\beta^{-n} \hat{h}^{-}(P) .
$$

Since $\beta>1$ and $\hat{h}^{+}(P)>0$, taking $n^{\text {th }}$-roots and letting $n \rightarrow \infty$ gives $\alpha_{\varphi}(P)=\beta=\delta_{\varphi}$.

\section{REFERENCES}

[1] N. Abarenkova, J.-Ch. Anglès d'Auriac, S. Boukraa, S. Hassani, and J.-M. Maillard. Topological entropy and Arnold complexity for two-dimensional mappings. Phys. Lett. A, 262(1):44-49, 1999.

[2] R. L. Adler, A. G. Konheim, and M. H. McAndrew. Topological entropy. Trans. Amer. Math. Soc., 114:309-319, 1965.

[3] Ekaterina Amerik. Existence of non-preperiodic algebraic points for a rational self-map of infinite order. Math. Res. Lett., 18(2):251-256, 2011.

[4] J.-Ch. Anglès d'Auriac, J.-M. Maillard, and C. M. Viallet. On the complexity of some birational transformations. J. Phys. A, 39(14):3641-3654, 2006.

[5] V. I. Arnol'd. Dynamics of complexity of intersections. Bol. Soc. Brasil. Mat. (N.S.), 21(1):1-10, 1990.

[6] Alan Baker. Transcendental number theory. Cambridge Mathematical Library. Cambridge University Press, Cambridge, second edition, 1990.

[7] Matthew Baker. A finiteness theorem for canonical heights attached to rational maps over function fields. J. Reine Angew. Math., 626:205-233, 2009.

[8] Eric Bedford and Kyounghee Kim. On the degree growth of birational mappings in higher dimension. J. Geom. Anal., 14(4):567-596, 2004.

[9] Eric Bedford and Kyounghee Kim. Degree growth of matrix inversion: birational maps of symmetric, cyclic matrices. Discrete Contin. Dyn. Syst., 21(4):977-1013, 2008.

[10] Eric Bedford and Kyounghee Kim. Linear recurrences in the degree sequences of monomial mappings. Ergodic Theory Dynam. Systems, 28(5):1369-1375, 2008 . 
[11] Eric Bedford, Kyounghee Kim, Tuyen Trung Truong, Nina Abarenkova, and Jean-Marie Maillard. Degree complexity of a family of birational maps. Math. Phys. Anal. Geom., 11(1):53-71, 2008.

[12] M. P. Bellon and C.-M. Viallet. Algebraic entropy. Comm. Math. Phys., 204(2):425-437, 1999.

[13] Robert L. Benedetto. Heights and preperiodic points of polynomials over function fields. Int. Math. Res. Not., (62):3855-3866, 2005.

[14] Enrico Bombieri and Walter Gubler. Heights in Diophantine Geometry. Number 4 in New Mathematical Monographs. Cambridge University Press, Cambridge, 2006.

[15] Sébastien Boucksom, Charles Favre, and Mattias Jonsson. Degree growth of meromorphic surface maps. Duke Math. J., 141(3):519-538, 2008.

[16] Alexandru Buium. Complex dynamics and invariant forms mod p. Int. Math. Res. Not., (31):1889-1899, 2005.

[17] Gregory S. Call and Joseph H. Silverman. Canonical heights on varieties with morphisms. Compositio Math., 89(2):163-205, 1993.

[18] Dan Coman. On the dynamics of a class of quadratic polynomial automorphisms of $\mathbb{C}^{3}$. Discrete Contin. Dyn. Syst., 8(1):55-67, 2002.

[19] Dan Coman and John Erik Fornæss. Green's functions for irregular quadratic polynomial automorphisms of $\mathbf{C}^{3}$. Michigan Math. J., 46(3):419-459, 1999.

[20] Laurent Denis. Points périodiques des automorphismes affines. J. Reine Angew. Math., 467:157-167, 1995.

[21] Dikran Dikranjan, Brendan Goldsmith, Luigi Salce, and Paolo Zanardo. Algebraic entropy for abelian groups. Trans. Amer. Math. Soc., 361(7):3401-3434, 2009.

[22] J. Diller and C. Favre. Dynamics of bimeromorphic maps of surfaces. Amer. J. Math., 123(6):1135-1169, 2001.

[23] Tien-Cuong Dinh and Nessim Sibony. Une borne supérieure pour l'entropie topologique d'une application rationnelle. Ann. of Math. (2), 161(3):1637$1644,2005$.

[24] Charles Favre and Mattias Jonsson. Eigenvaluations. Ann. Sci. École Norm. Sup. (4), 40(2):309-349, 2007.

[25] Charles Favre and Mattias Jonsson. Dynamical compactifications of $\mathbf{C}^{2}$. Ann. of Math. (2), 173(1):211-248, 2011.

[26] Charles Favre and Elizabeth Wulcan. Degree growth of monomial maps and McMullen's polytope algebra, 2010. Indiana Math. J., to appear.

[27] John Erik Fornaess and Nessim Sibony. Complex dynamics in higher dimension. II. In Modern Methods in Complex Analysis (Princeton, NJ, 1992), volume 137 of Ann. of Math. Stud., pages 135-182. Princeton Univ. Press, Princeton, NJ, 1995.

[28] D. Ghioca and T. J. Tucker. Periodic points, linearizing maps, and the dynamical Mordell-Lang problem. J. Number Theory, 129(6):1392-1403, 2009.

[29] Dragos Ghioca, Thomas J. Tucker, and Michael E. Zieve. Intersections of polynomials orbits, and a dynamical Mordell-Lang conjecture. Invent. Math., 171(2):463-483, 2008.

[30] Anna Giordano Bruno and Simone Virili. Algebraic Yuzvinski formula, 2011. arXiv:1111.1287. 
[31] B. Grammaticos, R. G. Halburd, A. Ramani, and C.-M. Viallet. How to detect the integrability of discrete systems. J. Phys. A, 42(45):454002, 30, 2009.

[32] R. G. Halburd. Diophantine integrability. J. Phys. A, 38(16):L263-L269, 2005.

[33] Boris Hasselblatt and James Propp. Degree-growth of monomial maps. Ergodic Theory Dynam. Systems, 27(5):1375-1397, 2007. Corrigendum vol. 6, page 1999.

[34] Marc Hindry and Joseph H. Silverman. Diophantine Geometry: An Introduction, volume 201 of Graduate Texts in Mathematics. Springer-Verlag, New York, 2000.

[35] A. N. W. Hone. Diophantine non-integrability of a third-order recurrence with the Laurent property. J. Phys. A, 39(12):L171-L177, 2006.

[36] Mattias Jonsson and Elizabeth Wulcan. Stabilization of monomial maps. Michigan Math. J., 60:629-660, 2011.

[37] Mattias Jonsson and Elizabeth Wulcan. Canonical heights for plane polynomial maps of small topological degree: A conjecture of Silverman, 2012. arXiv: 1202.0203.

[38] Shu Kawaguchi. Canonical height functions for affine plane automorphisms. Math. Ann., 335(2):285-310, 2006.

[39] Shu Kawaguchi. Local and global canonical height functions for affine space regular automorphisms, 2009. arXiv:0909.3573.

[40] Shu Kawaguchi and Joseph H. Silverman. On the dynamical and arithmetic degrees of rational self-maps of algebraic varieties, 2012. arXiv:1208.0815.

[41] Serge Lang. Fundamentals of Diophantine Geometry. Springer-Verlag, New York, 1983.

[42] ChongGyu Lee. An upper bound for the height for regular affine automorphisms of $\mathbb{A}^{n}, 2009$. arXiv:0909.3107, Mathematische Annalen, to appear.

[43] Jan-Li Lin. Pulling back cohomology classes and dynamical degrees of monomial maps, 2010. arXiv:1010.6285, to appear in Bull. SMF.

[44] Jan-Li Lin. Algebraic stability and degree growth of monomial maps. Math. Z., 271(1-2):293-311, 2012.

[45] Kazutoshi Maegawa. Quadratic polynomial automorphisms of dynamical degree golden ratio of $\mathbb{C}^{3}$. Ergodic Theory Dynam. Systems, 21(3):823-832, 2001.

[46] Sandra Marcello. Sur la dynamique arithmétique des automorphismes de l'espace affine. Bull. Soc. Math. France, 131(2):229-257, 2003.

[47] Viêt-Anh Nguyên. Algebraic degrees for iterates of meromorphic self-maps of $\mathbb{P}^{k}$. Publ. Mat., 50(2):457-473, 2006.

[48] Justin Peters. Entropy on discrete abelian groups. Adv. in Math., 33(1):1-13, 1979.

[49] Justin Peters. Entropy of automorphisms on L.C.A. groups. Pacific J. Math., 96(2):475-488, 1981.

[50] John A. G. Roberts and Franco Vivaldi. Arithmetical method to detect integrability in maps. Phys. Rev. Lett., 90(3):034102, 4, 2003.

[51] John A. G. Roberts and Franco Vivaldi. Signature of time-reversal symmetry in polynomial automorphisms over finite fields. Nonlinearity, 18(5):2171-2192, 2005.

[52] John A. G. Roberts and Franco Vivaldi. A combinatorial model for reversible rational maps over finite fields. Nonlinearity, 22(8):1965-1982, 2009. 
[53] Alexander Russakovskii and Bernard Shiffman. Value distribution for sequences of rational mappings and complex dynamics. Indiana Univ. Math. J., 46(3):897-932, 1997.

[54] Nessim Sibony. Dynamique des applications rationnelles de $\mathbb{P}^{k}$. In Dynamique et géométrie complexes (Lyon, 1997), volume 8 of Panor. Synthèses, pages ix-x, xi-xii, 97-185. Soc. Math. France, Paris, 1999.

[55] Joseph H. Silverman. Rational points on $K 3$ surfaces: a new canonical height. Invent. Math., 105(2):347-373, 1991.

[56] Joseph H. Silverman. Geometric and arithmetic properties of the Hénon map. Math. Z., 215(2):237-250, 1994.

[57] Joseph H. Silverman. The Arithmetic of Dynamical Systems, volume 241 of Graduate Texts in Mathematics. Springer, New York, 2007.

[58] Joseph H. Silverman. The Arithmetic of Elliptic Curves, volume 106 of Graduate Texts in Mathematics. Springer, Dordrecht, second edition, 2009.

[59] Tuyen Trung Truong. Degree complexity of a family of birational maps. II. Exceptional cases. Math. Phys. Anal. Geom., 12(2):157-180, 2009.

[60] Simone Virili. Entropy for endomorphisms of LCA groups. Topology Appl., 159(9):2546-2556, 2012.

[61] Junyi Xie. Periodic points of birational maps on the projective plane, 2011. arXiv:1106.1825.

\section{Appendix A. AdDitional material}

In this appendix we give further details and comments regarding various statements in the body of the article. This appendix is for the ArXiv version of this article; it will not appear in the published version.

\section{A.1. Description of the divisible hull.}

Let $G$ be an algebraic subgroup of $\mathbb{G}_{m}^{N}$. We verify that $G(\overline{\mathbb{Q}})^{\text {div }}=$ $G(\overline{\mathbb{Q}}) \mathbb{G}_{m}^{N}(\overline{\mathbb{Q}})_{\text {tors }}$.

Let $Q \in G(\overline{\mathbb{Q}})$ and $\zeta \in \mathbb{G}_{m}^{N}(\overline{\mathbb{Q}})_{\text {tors. }}$. Choose an $n \geq 1$ such that $\boldsymbol{\zeta}^{n}=1$. Write $G=G_{L}$ for a lattice $L \subset \mathbb{Z}^{N}$, i.e., with the obvious notation, the group $G$ is the set of points satisfying $P^{\mathbf{e}}$ for every $\mathbf{e} \in L$. Then every e $\in L$ we have

$$
\left((\boldsymbol{\zeta} Q)^{n}\right)^{\mathbf{e}}=\left(\boldsymbol{\zeta}^{n}\right)^{\mathbf{e}}\left(Q^{\mathbf{e}}\right)^{n}=1 \cdot 1=1,
$$

which proves that $\zeta Q \in G(\overline{\mathbb{Q}})^{\text {div }}$.

For the converse, we suppose that $P \in G_{L}(\mathbb{C})^{\text {div }}$, say $P^{n} \in G_{L}(\mathbb{C})$ for some $n \geq 1$. Thus $P^{n \mathbf{e}}=1$ for all $\mathbf{e} \in L$, so $P^{\mathbf{e}} \in \boldsymbol{\mu}_{n}$ for all $\mathbf{e} \in L$. In this way we get a homomorphism

$$
\xi: L \longrightarrow \boldsymbol{\mu}, \quad \xi_{\mathbf{e}}=P^{\mathbf{e}}
$$

We want to prove that there is an element $\boldsymbol{\zeta} \in \boldsymbol{\mu}^{N}$ with the property that $\boldsymbol{\zeta}^{\mathbf{e}}=P^{\mathbf{e}}$ for all $\mathbf{e} \in L$, since then $\boldsymbol{\zeta}^{-1} P \in G_{L}(\mathbb{C})$ and $\zeta \in \mathbb{G}_{m}^{N}(\mathbb{C})_{\text {tors }}$. 
Notationally it's easier if we identify $\mathbf{u}$ with $\mathbb{Q} / \mathbb{Z}$ via the map $t \mapsto$ $e^{2 \pi i t}$. Then $\xi$ is a homomorphism

$$
\xi \in \operatorname{Hom}(L, \mathbb{Q} / \mathbb{Z})
$$

and we want to know if $\xi$ lifts to an element of $\operatorname{Hom}\left(\mathbb{Z}^{N}, \mathbb{Q} / \mathbb{Z}\right) \cong$ $\operatorname{Hom}(\mathbb{Q} / \mathbb{Z})^{N}$. In other words, we want to know if the map

$$
\operatorname{Hom}\left(\mathbb{Z}^{N}, \mathbb{Q} / \mathbb{Z}\right) \longrightarrow \operatorname{Hom}(L, \mathbb{Q} / \mathbb{Z})
$$

induced by $L \subset \mathbb{Z}^{N}$ is surjective. Letting $K=\mathbb{Z}^{N} / L$, this is equivalent to showing that $\operatorname{Ext}^{1}(K, \mathbb{Q} / \mathbb{Z})=0$. Since $K$ is a direct sum of cyclic groups $C_{m}$ and copies of $\mathbb{Z}$, it suffices to prove that

$$
\operatorname{Ext}^{1}\left(C_{m}, \mathbb{Q} / Z Z\right)=\operatorname{Ext}^{1}(\mathbb{Z}, \mathbb{Q} / Z Z)=0 .
$$

Applying $\operatorname{Hom}(\cdot, \mathbb{Q} / \mathbb{Z})$ to the exact sequence $0 \rightarrow \mathbb{Z} \stackrel{m}{\rightarrow} \mathbb{Z} \rightarrow C_{m} \rightarrow 0$, we see that

$$
\operatorname{Ext}^{1}\left(C_{m}, \mathbb{Q} / Z Z\right)=\operatorname{Ext}^{1}(\mathbb{Z}, \mathbb{Q} / Z Z)[m],
$$

so we are reduced to proving that $\operatorname{Ext}^{1}(\mathbb{Z}, \mathbb{Q} / Z Z)=0$. But $\mathbb{Z}$ is projective, so $\operatorname{Ext}^{1}(\mathbb{Z}, A)=0$ for any abelian group.

\section{A.2. Proof of Lemma 34(d).}

We first prove that

$$
\operatorname{Perp}_{F}\left(V_{1} \dot{+} \cdots \dot{+} V_{t}\right)=\operatorname{Perp}_{F}\left(V_{1}\right) \cap \cdots \cap \operatorname{Perp}_{F}\left(V_{t}\right) .
$$

Let $\mathbf{w} \in \operatorname{Perp}_{F}\left(V_{1} \dot{+} \cdots \dot{+} V_{t}\right)$. Then $\mathbf{w}$ certainly annihilates every $V_{i}$, so $\mathbf{v} \in \operatorname{Perp}\left(V_{i}\right)$ for all $i$, and hence $\mathbf{v} \in \operatorname{Perp}_{F}\left(V_{1}\right) \cap \cdots \cap \operatorname{Perp}_{F}\left(V_{t}\right)$. This proves that

$$
\operatorname{Perp}_{F}\left(V_{1} \dot{+} \cdots \dot{+} V_{t}\right) \subset \operatorname{Perp}_{F}\left(V_{1}\right) \cap \cdots \cap \operatorname{Perp}_{F}\left(V_{t}\right) .
$$

Next let $\mathbf{u} \in \operatorname{Perp}_{F}\left(V_{1}\right) \cap \cdots \cap \operatorname{Perp}_{F}\left(V_{t}\right)$, and let $\mathbf{v} \in V_{1} \dot{+} \cdots \dot{+} V_{t}$. Then $\mathbf{v}=\mathbf{v}_{1}+\cdots+\mathbf{v}_{N}$ with $\mathbf{v}_{i} \in V_{i}$. But $\mathbf{u} \in \operatorname{Perp}_{F}\left(V_{i}\right)$ for all $i$, so $\mathbf{u} \cdot \mathbf{v}_{i}=0$ for all $i$, so $\mathbf{u} \cdot \mathbf{v}=0$. Hence $\mathbf{u} \in \operatorname{Perp}_{F}\left(V_{1} \dot{+} \cdots \dot{+} V_{t}\right)$, which proves the other inclusion

$$
\operatorname{Perp}_{F}\left(V_{1}\right) \cap \cdots \cap \operatorname{Perp}_{F}\left(V_{t}\right) \subset \operatorname{Perp}_{F}\left(V_{1} \dot{+} \cdots \dot{+} V_{t}\right) .
$$

This proves (42).

We next use (42), replacing $V_{i}$, with $\operatorname{Perp}_{F}\left(V_{i}\right)$, and use (c) to delete double perps. This gives

$$
\begin{aligned}
V_{1} \cap \cdots \cap V_{t} & =\operatorname{Perp}_{F}\left(\operatorname{Perp}_{F}\left(V_{1}\right)\right) \cap \cdots \cap \operatorname{Perp}_{F}\left(\operatorname{Perp}_{F}\left(V_{t}\right)\right) \quad \text { from }(\mathrm{c}), \\
& =\operatorname{Perp}_{F}\left(\operatorname{Perp}_{F}\left(V_{1}\right) \dot{+} \cdots+\operatorname{Perp}_{F}\left(V_{t}\right)\right) \quad \text { from }(42) .
\end{aligned}
$$

Applying $\operatorname{Perp}_{F}$ to this equality and using (c) again gives

$$
\operatorname{Perp}_{F}\left(V_{1} \cap \cdots \cap V_{t}\right)=\operatorname{Perp}_{F}\left(V_{1}\right) \dot{+} \cdots \dot{+} \operatorname{Perp}_{F}\left(V_{t}\right),
$$


which is the desired result.

\section{A.3. Baker's theorem.}

We prove that

$$
\operatorname{Perp}_{\overline{\mathbb{Q}}}(\mathbf{w}) \cong \operatorname{Perp}_{\mathbb{Q}}(\mathbf{w}) \otimes_{\mathbb{Q}} \overline{\mathbb{Q}}
$$

by induction on the dimension $k$ of $\operatorname{Perp}_{\overline{\mathbb{Q}}}(\mathbf{w})$. The result is trivial if $k=0$, and as already noted, the case $k=1$ is the classical statement of Baker's theorem. Assume now that we know the result for $k$, and let $\operatorname{dim} \operatorname{Perp}_{\overline{\mathbb{Q}}}(\mathbf{w})=k+1$. Write generators for the relations in $\operatorname{Perp}_{\overline{\mathbb{Q}}}(\mathbf{w})$ as the rows of a $(k+1)$-by- $N$ matrix $B$, so we have $B \mathbf{w}=0$. Permuting the rows of $B$ and the coordinates of $\mathbf{w}$, we may assume that $b_{k+1, N} \neq 0$, and then subtracting multiples of the last row of $B$ from the other rows, we may assume that $b_{i N}=0$ for $1 \leq i \leq k$. We now let

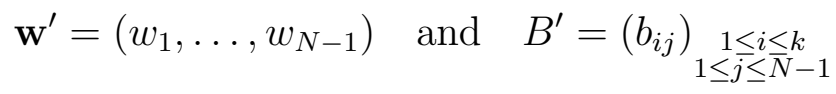

Then the rows of $B^{\prime}$ generate $\operatorname{Perp}_{\overline{\mathbb{Q}}}\left(\mathbf{w}^{\prime}\right)$, so by the induction hypothesis, the space $\operatorname{Perp}_{\overline{\mathbb{Q}}}\left(\mathbf{w}^{\prime}\right)$ has a basis in $\operatorname{Perp}_{\mathbb{Q}}(\mathbf{w})$. This means that we can replace $B^{\prime} \in \operatorname{Mat}_{k \times N}(\overline{\mathbb{Q}})$ with a matrix in $\operatorname{Mat}_{k \times N}(\mathbb{Q})$, and hence we may assume that the first $k$ rows of $B$ have coefficients in $\mathbb{Q}$ (and the final entry in each of these rows is 0 ).

We now repeat the argument with a different row and column. The first row of $B$ must have a non-zero entry (and note that the last entry is zero), so relabeling the first $N-1$ coordinates of $\mathbf{w}$, we may assume that $b_{11} \neq 0$. Subtracting multiples of the first row from the other rows, we may further assume that $b_{i 1}=0$ for all $2 \leq i \leq k+1$. We let

$$
\mathbf{w}^{\prime \prime}=\left(w_{2}, \ldots, w_{N}\right) \quad \text { and } \quad B^{\prime \prime}=\left(b_{i j}\right)_{\substack{2 \leq i \leq k+1 \\ 2 \leq j \leq N}},
$$

so the rows of $B^{\prime \prime}$ generate $\operatorname{Perp}_{\overline{\mathbb{Q}}}\left(\mathbf{w}^{\prime \prime}\right)$. Again by the induction hy-

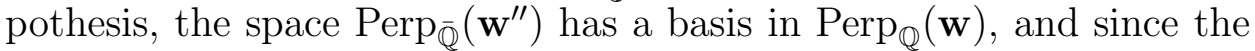
last column of $B^{\prime \prime}$ is not zero (since $b_{k+1, N} \neq 0$ ), there must be some vector $\left(c_{2}, \ldots, c_{N}\right) \in \operatorname{Perp}_{\mathbb{Q}}\left(\mathbf{w}^{\prime \prime}\right)$ with $c_{N} \neq 0$. This vector is not in the $\mathbb{Q}$-span of the first $k$ rows of $B^{\prime \prime}$, since the last coordinates of the first $k$ rows of $B^{\prime \prime}$ are all zero. Hence the vector

$$
\mathbf{c}=\left(0, c_{2}, \ldots, c_{N}\right)
$$

is in $\operatorname{Perp}_{\mathbb{Q}}(\mathbf{w})$ and is not in the span of the first $k$ rows of $B$. This proves that the first $k$ rows of $B$ and the vector c generate a $\mathbb{Q}$-vector subspace of $\operatorname{Perp}_{\mathbb{Q}}(\mathbf{w})$ of dimension $k+1$, which is equal to the dimension of $\operatorname{Perp}_{\overline{\mathbb{Q}}}(\mathbf{w})$.

Amusing remark: There are many contrived examples of incorrect induction proofs in which the case $k=0$ is easy, and if $k \geq 1$, then the proof from $k$ to $k+1$ is easy, but one glosses over the fact that 
the induction argument is incorrect when one tries to go from $k=0$ to $k=1$. The above proof has this form, i.e., $k=0$ is easy, and $k$ implies $k+1$ is easy for $k \geq 1$. Of course, the full proof is correct because $k=1$ is also true, but the $k=1$ case is not provable by a trivial induction from the $k=0$ case. Indeed, as noted, the case $k=1$ is the qualitative statement of Baker's linear forms in logarithms theorem.

A.4. Proof of inequality (19).

Let $B=\left(\beta_{i j}\right)$ and $P=\left(x_{1}, \ldots, x_{N}\right)$. Then

$$
\begin{aligned}
\sum_{v \in M_{K}} \max B \log \|P\|_{v} & =\sum_{v \in M_{K}} \max _{1 \leq i \leq N}\left\{\sum_{j=1}^{N} \beta_{i j} \log \left\|x_{j}\right\|_{v}\right\} \\
& \geq \max _{1 \leq i \leq N}\left\{\sum_{v \in M_{K}}\left(\sum_{j=1}^{N} \beta_{i j} \log \left\|x_{j}\right\|_{v}\right)\right\} \\
& \geq \max _{1 \leq i \leq N}\left\{\sum_{j=1}^{N} \beta_{i j}\left(\sum_{v \in M_{K}} \log \left\|x_{j}\right\|_{v}\right)\right\} \\
& =0,
\end{aligned}
$$

where the first inequality is due to the fact that a sum of maxs may be strictly larger than the max of the sum, and where the last equality follows from the product formula. (Note that all of the $x_{j}$ are nonzero by assumption, since $P \in \mathbb{G}_{m}^{N}(\overline{\mathbb{Q}})$.

A.5. Description of $W_{1} \dot{+} \cdots \dot{+} W_{r} \dot{+} Z$ as a kernel. The space $W_{1} \dot{+} \cdots \dot{+} W_{r} \dot{+} Z$ is the kernel of the matrix

$$
\prod_{|\lambda|<\rho}(A-\lambda)^{N} \cdot \prod_{|\lambda|=\rho}(A-\lambda)^{\ell} \in \operatorname{Mat}_{N}(\overline{\mathbb{Q}}) .
$$

This follows from the fact if $V \subset \overline{\mathbb{Q}}^{N}$ is a Jordan subspace for $A$ with eigenvalue $\lambda$, then:

- If $|\lambda|<\rho$, then $(A-\lambda)^{N}$ annihilates $V$.

- If $|\lambda|=\rho$ and $\operatorname{dim} V \leq \ell$, then $(A-\lambda)^{\ell}$ annihilates $V$.

- If $|\lambda|=\rho$ and $\operatorname{dim} V=\ell$, so $V$ is maximal, then $(A-\lambda)^{\ell}$ annihilates $W$, while $(A-\lambda)^{\ell}$ acts as a nonzero scalar on $V / W$; cf. (24).

\section{A.6. The smallest group containing a $\varphi$-orbit is $\varphi$-invariant.}

Let $P \in \mathbb{G}_{m}^{N}(\mathbb{C})$. The claim is that if $G \subset \mathbb{G}_{m}^{N}$ the the smallest algebraic subgroup of $\mathbb{G}_{m}^{N}$ containing the orbit $\mathcal{O}_{\varphi}(P)$, then $\varphi(G) \subset G$. We remark that the proof works more generally if $\mathbb{G}_{m}^{N}$ is replaced by any (commutative) algebraic group $A$ (over a field of characteristic 0 ). 
So we let $A$ be such a group, let $\varphi: A \rightarrow A$ be an algebraic homomorphism, and let $\alpha \in A(\mathbb{C})$ be a point. For each $n \geq 1$, let $H_{n} \subset A$ be the Zariski closure of the subgroup of $A$ generated by $\varphi^{n}(\alpha)$,

$$
H_{n}=\overline{\left\{\varphi^{n}(\alpha)^{k}: k \in \mathbb{Z}\right\}}
$$

Then $H_{n}$ is a Zariski closed subset of $A$. Further, the fact that $H_{n}$ is the closure of a subgroup implies that $H_{n}$ is closed under the group law, so $H_{n}$ is an algebraic subgroup of $A$.

The group $G$ contains $\mathcal{O}_{\varphi}(\alpha)$ by assumption, and its closed and a group, so it contains all of the $H_{n}$. We claim that $G$ is the smallest algebraic subgroup containing all of the $H_{n}$. To see this, suppose that $G^{\prime}$ is an algebraic subgroup and $G \supset H_{n}$ for all $n \geq 0$. Since $\varphi^{n}(\alpha) \in H_{n}$, this implies in particular that $\varphi^{n}(\alpha) \in G^{\prime}$, and since this holds for all $n \geq 0$, we see that $\mathcal{O}_{\varphi}(\alpha) \subset G^{\prime}$. But $G$ is the smallest algebraic subgroup containing $\mathcal{O}_{\varphi}(\alpha)$, so $G \subset G^{\prime}$. This proves the claim.

We now consider a chain of containments:

$$
\begin{array}{rlrl}
G \supset G \backslash H_{0} & \supset \bigcup_{n \geq 1} H_{n} & & \text { since } G \text { contains } \bigcup_{n \geq 0} H_{n}, \\
& =\bigcup_{n \geq 0} \varphi\left(H_{n}\right) & & \text { since } \varphi\left(H_{n}\right)=H_{n+1}, \\
& =\varphi\left(\bigcup_{n \geq 0} H_{n}\right) . &
\end{array}
$$

Hence

$$
\varphi^{-1}(G) \supset \bigcup_{n \geq 0} H_{n}
$$

But $\varphi^{-1}(G)$ is an algebraic subgroup of $A$, while we showed earlier that $G$ is the smallest algebraic subgroup of $A$ that contains $\bigcup_{n \geq 0} H_{n}$. Hence $G \subset \varphi^{-1}(G)$, which gives the desired inclusion $\varphi(G) \subset G$.

We remark that it is possible for $\varphi(G)$ to be strictly contained in $G$. For example, this happens if $G$ has more than one connected component and $\varphi(G)$ is contained in the identity component of $G$.

\section{A.7. Arithmetic degree independent of ambient space.}

We claimed that if $i: \mathbb{P}^{k} \rightarrow \mathbb{P}^{N}$ with $\operatorname{dim} i\left(\mathbb{P}^{k}\right)=k$, then $h_{\mathbb{P}^{N}} \circ i \asymp h_{\mathbb{P}^{k}}$. Assuming this, consider any map $\varphi: \mathbb{P}^{N} \rightarrow \mathbb{P}^{N}$ that descends to a $\operatorname{map} \psi: \mathbb{P}^{k} \rightarrow \mathbb{P}^{k}$, i.e., so that $i \circ \psi=\varphi \circ i$. Then for any $P \in \mathbb{P}^{k}(\overline{\mathbb{Q}})$ we have

$$
h_{\mathbb{P}^{N}}\left(\varphi^{n}(i(P))\right)=h_{\mathbb{P}^{N}}\left(i\left(\psi^{n}(P)\right)\right) \asymp h_{\mathbb{P}^{k}}\left(\psi^{n}(P)\right) .
$$


Taking $n^{\text {th }}$-roots and the limsup as $n \rightarrow \infty$, we see that $\alpha_{\varphi}(i(P))=$ $\alpha_{\psi}(P)$, i.e., we get the same value for the arithmetic degree regardless of where we do the computation.

The claim is easy, since $i$ satisfies $i^{*} \mathcal{O}_{\mathbb{P}^{N}}(1)=\mathcal{O}_{\mathbb{P}^{k}}(m)$ for some $m \geq$ 1. (These are line bundles, not orbits.) It follows from standard properties of heights that $h_{\mathbb{P}^{N}} \circ i=m h_{\mathbb{P}^{k}}+O(1)$, which is stronger than what we claimed.

A.8. A Jordan block condition that implies $\alpha_{\varphi}(P)<\delta_{\varphi}$.

We proved that if $A$ is diagonalizable, then $\alpha_{\varphi}(P)<\delta_{\varphi}$. A variation of the same argument can be used to prove the implication

$$
\hat{h}_{\varphi}(P)=0 \Longrightarrow \alpha_{\varphi}(P)<\delta_{\varphi}
$$

under the weaker hypothesis

$$
\left(\begin{array}{l}
\text { Every Jordan subspace for } A \text { whose eigenvalue } \lambda \\
\text { satisfies }|\lambda|=\rho(A) \text { is a maximal Jordan subspace }
\end{array}\right) \text {. }
$$

Note that since the Jordan blocks of a diagonalizable matrix have dimension 1 , such matrices clearly satisfy $(*)$.

\section{A.9. Verification of the implication in Remark 38.}

If $\hat{h} \asymp h$ and $\hat{h}_{\varphi}(P)=0$, then

$$
\begin{aligned}
h\left(\varphi^{n}(P)\right) & \asymp \hat{h}\left(\varphi^{n}(P)\right) \\
& =\hat{h}_{\varphi}\left(\varphi^{n}(P)\right)+\hat{h}_{\varphi^{-1}}\left(\varphi^{n}(P)\right) \\
& =\delta_{\varphi}^{n} \hat{h}_{\varphi}(P)+\delta_{\varphi^{-1}}^{-n} \hat{h}_{\varphi^{-1}}(P) \\
& =\delta_{\varphi^{-1}}^{-n} \hat{h}_{\varphi^{-1}}(P) .
\end{aligned}
$$

Thus $\left\{\varphi^{n}(P)\right\}$ is a set of bounded height, hence finite, so $P$ is periodic.

E-mail address: jhs@math.brown.edu

Mathematics Department, Box 1917 Brown University, Providence, RI 02912 USA 